SANDIA REPORT

SAND97-2860 • UC-1420

Unlimited Release

Printed November 1997
RECEIVEO

DEC 151997

OSTI

9

\title{
Development and Testing of an Active Boring Bar for Increased Chatter Immunity
}

Jim Redmond, Pat Barney

Prepared by

Sandia National Laboratories

Albuquerque, New Mexico 87185 and Livermore, California 94550

Sandia is a multiprogram laboratory operated by Sandia Corporation, a Lockheed Martin Company, for the United States Department of Energy under Contract DE-AC04-94AL85000.

Approved for public release; further dissemination unlimited.

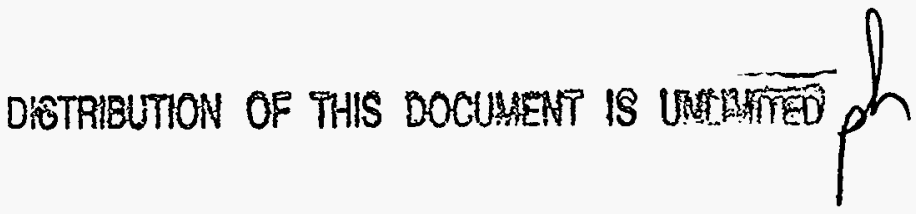

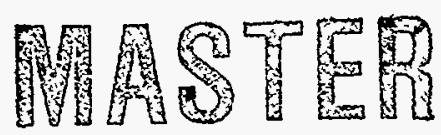

\section{Sandia National Laboratories}


Issued by Sandia National Laboratories, operated for the United States Department of Energy by Sandia Corporation.

NOTICE: This report was prepared as an account of work sponsored by an agency of the United States Government. Neither the United States Government nor any agency thereof, nor any of their employees, nor any of their contractors, subcontractors, or their employees, makes any warranty, express or implied, or assumes any legal liability or responsibility for the accuracy, completeness, or usefulness of any information, apparatus, product, or process disclosed, or represents that its use would not infringe privately owned rights. Reference herein to any specific commercial product, process, or service by trade name, trademark, manufacturer, or otherwise, does not necessarily constitute or imply its endorsement, recommendation, or favoring by the United States Government, any agency thereof, or any of their contractors or subcontractors. The views and opinions expressed herein do not necessarily state or reflect those of the United States Government, any agency thereof, or any of their contractors.

Printed in the United States of America. This report has been reproduced directly from the best available copy.

Available to DOE and DOE contractors from

Office of Scientific and Technical Information

P.O. Box 62

Oak Ridge, TN 37831

Prices available from (615) 576-8401, FTS 626-8401

Available to the public from

National Technical Information Service

U.S. Department of Commerce

5285 Port Royal Rd

Springfield, VA 22161

NTIS price codes

Printed copy: A03

Microfiche copy: A01 


\section{DISCLATMER}

Portions of this doecment may be illegible in electronic image produets. Images are produced from the best available oxiginal documentert. 


\title{
Development and Testing of an Active Boring Bar for Increased Chatter Immunity
}

\author{
Jim Redmond ${ }^{1}$ \\ Structural Dynamics and Vibration Control Department \\ and \\ Pat Barney ${ }^{2}$ \\ Experiment Structural Dynamics Department
}

\author{
Sandia National Laboratories \\ P.O. Box 5800 \\ Albuquerque, NM 87185-0439
}

\begin{abstract}
Recent advances in smart materials have renewed interest in the development of improved manufacturing processes featuring sensing, processing, and active control. In particular, vibration suppression in metal cutting has received much attention because of its potential for enhancing part quality while reducing the time and cost of production. Although active tool clamps have been recently demonstrated, they are often accompanied by interfacing issues that limit their applicability to specific machines.

Under the auspices of the Laboratory Directed Research and Development program, the project titled "Smart Cutting Tools for Precision Manufacturing" developed an alternative approach to active vibration control in machining. Using the boring process as a vehicle for exploration, a commercially available tool was modified to incorporate PZT stack actuators for active suppression of its bending modes. Since the modified tool requires no specialized mounting hardware, it can be readily mounted on many machines. Cutting tests conducted on a horizontal lathe fitted with a hardened steel workpiece verify that the actively damped boring bar yields significant vibration reduction and improved surface finishes as compared to an unmodified tool.
\end{abstract}

1. Mailstop: 0439, Phone: (505) 844-3136, Fax: (505) 844-9297, Email: jmredmo@sandia.gov

2. Mailstop: 0557, Phone: (505) 844-7689, Fax: (505) 844-0078, Email: psbarne@sandia.gov 
This page intentionally left blank. 


\section{Acknowledgments}

Throughout its two year duration, many people made valuable contributions toward the eventual success of this project. Andy Hazelton at Sandia Livermore (now of Nikon) facilitated the initial cutting tests that provided key insight into the phenomenon of chatter in boring. Andy Jojola designed the specialized interface for mounting the PZT stacks to the boring bar. David Smith of the University of Florida joined the project as a summer intern and provided critical input into the prototype design described in section 4 of this report. The evaluation of the prototype bar was conducted at Sandia Albuquerque in building 840 under the supervision of Charlie Salazar and Keith Mote. The success of the testing program was due in large part to the machining expertise provided by Terry Litts and Tim Mitchell. In addition to the contributions of these Sandians, the generous support of the LDRD program is gratefully acknowledged. Joe Kamman and Paul McCalmont of Cincinatti Milacron made a few trips to Sandia to provide no-cost assessments of the project from an industry perspective. We greatly appreciate their insightful evaluations and suggestions which helped to keep the project grounded in reality. We hope to continue this interaction on future projects. Finally, many thanks to Dan Segalman and Ron Rodeman of Sandia for their careful review of this report. 
This page intentionally left blank. 


\section{Table of Contents}

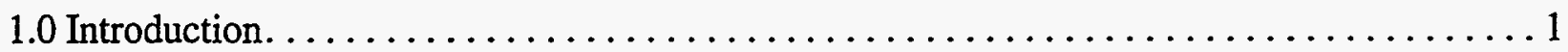

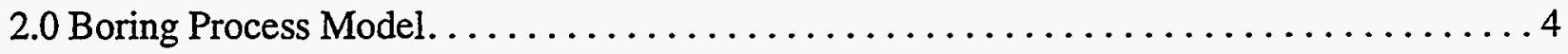

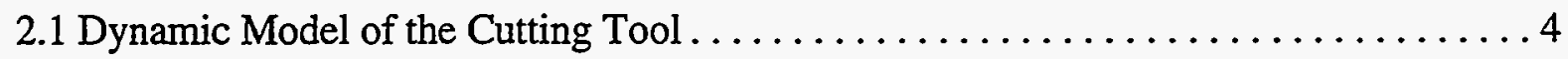

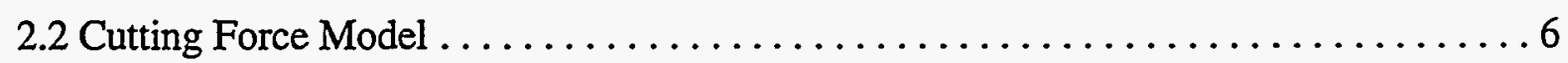

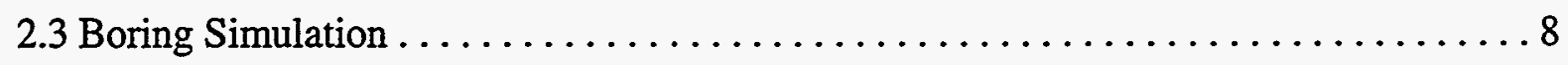

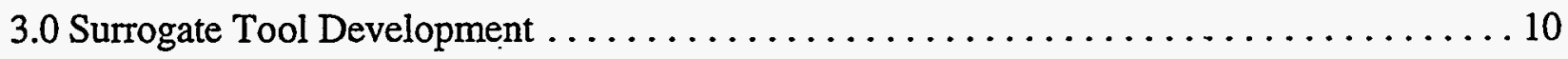

3.1 Actuation Methodology ................................... 10

3.2 Surrogate Tool Design. ................................. 13

3.3 Static Stiffness Assessment .............................. 13

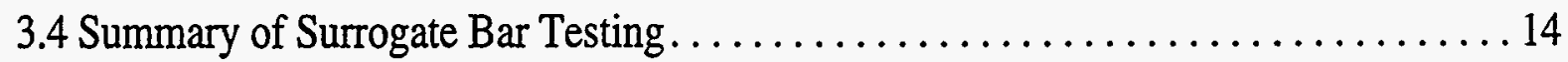

3.5 Example Simulation Based Evaluation ........................ 14

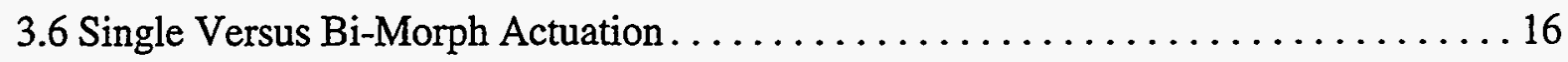

4.0 Prototype Design. . . . . . . . . . . . . . . . . . . . . . . . . 19

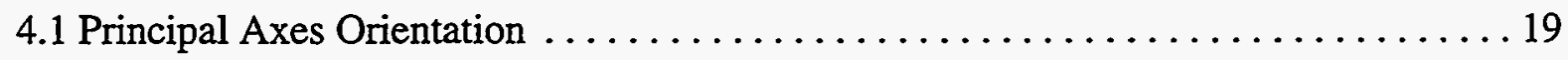

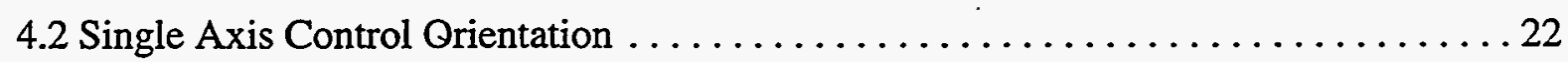

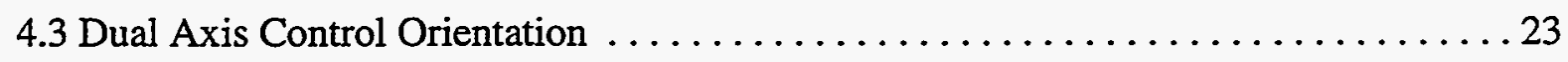

4.4 Prototype Design Recommendations. .......................... 24

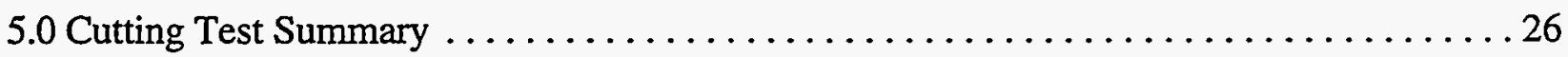

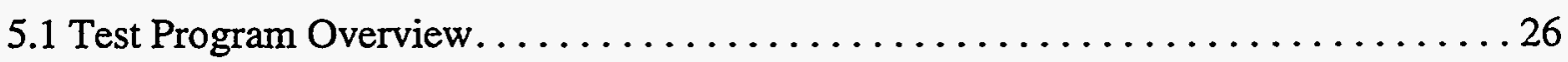

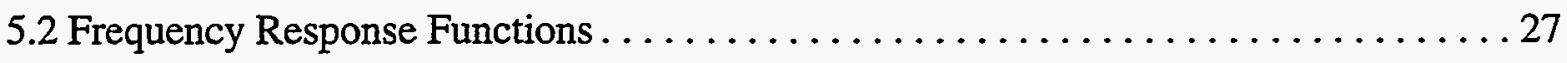

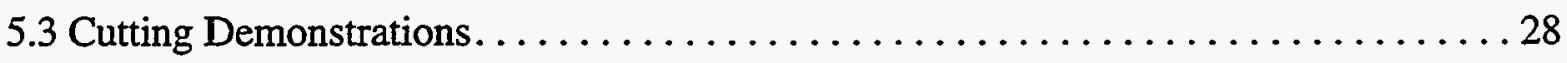

5.3.1 Depth of Cut 0.005 inches ............................ 29

5.3.2 Depth of Cut 0.010 inches ............................ 29

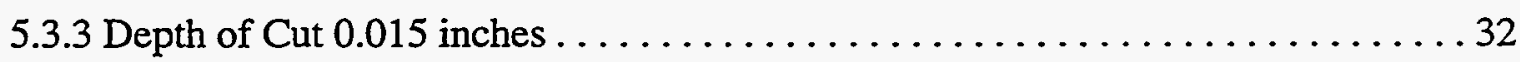

5.3.4 Depth of Cut 0.020 inches ............................. 33

5.4 Performance Assessment. . . . . . . . . . . . . . . . . . . . . . . . 34

6.0 Concluding Remarks...................................... 37

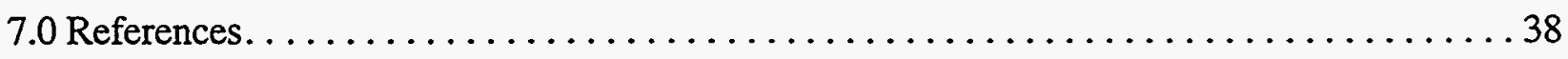

Appendix: Publications Associated with The Smart Tool LDRD ................ 40 
This page intentionally left blank. 
Request: ps2-107 from sass2247a

Options: flist='/tmp/boring_SAND.frm.ps.G600f'

Title: $107-1$

Wed Nov 19 15:50:08 MST 1997

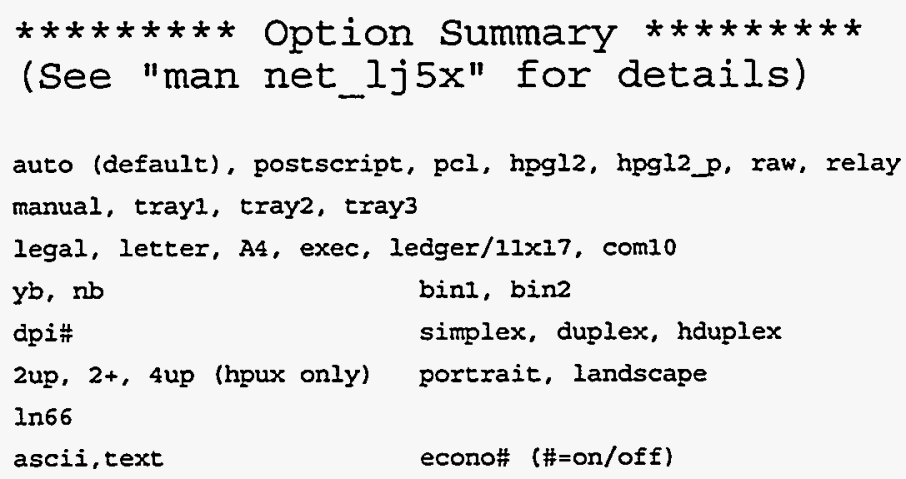




\subsection{Introduction}

This report summarizes the "Smart Cutting Tools for Precision Manufacturing" Laboratory Directed Research and Development project that was funded through the Advanced Manufacturing program area for FY96-97. The goal of the project was to demonstrate increased metal removal rate through the integration of smart material actuators with a conventional tool. In all metal cutting processes, the metal removal rate (MRR) is limited by the phenomenon of chatter (Ref. 1), a regenerative vibration that is driven by workpiece surface undulations resulting from previous tool vibrations. Because resistance to chatter in the absence of workpiece flexibility is a function of the damping inherent to the tool structure, in many cases the use of feedback control to stabilize the primary modes of vibration should permit substantially higher metal removal rates.

To facilitate the development of a smart materials based active control system for chatter mitigation, the boring process was selected as the featured Hazeltonmachining process of this research. This selection enabled project emphasis to be placed on modeling and control of the cutting process without considering the hardware complexities associated with sensing and actuating rotating tools. These hardware issues are currently being considered in an effort to extend this technology to rotating tool processes (Ref. 2).

Assuming a rigid workpiece, the precision that can be achieved in a given boring operation is determined by the static stiffness of the boring bar. Furthermore, the process stability is governed in large part by the bar's dynamic stiffness (Ref. 3), which can be thought of as a combination of the static stiffness and the inherent structural damping. Consequently, from both a precision and a stability viewpoint, it is desirable to use the stoutest tool available for a given operation. Many boring applications, however, necessitate the use of boring bars with high overhang ratios (L/D) for which it is difficult to achieve stability in the cutting process due to the inherent flexibility of the tool. This problem is regularly encountered in the manufacture of penetrating weapons which require internal contouring of the weapon system housing at very high overhang ratios.

Active and passive vibration absorbers tuned to a specific operating condition can provide relief in some boring operations. Most effective when placed in regions of high displacement near the bar tip (Refs. 4-5), these devices can provide vibration suppression in only one direction, typically normal or tangential to the workpiece surface. Furthermore, complications associated with cutting zone interference and coolant application make these approaches cumbersome in practice. Kennametal's recently introduced 'Tuned Tooling' product line featuring internally mounted electronically tuned passive dampers overcomes many of the problems of conventional passive dampers. These devices augment cutting stability by suppressing the tool vibration at the first bending mode frequency, which is determined by a pre-cut impact test. But because frequencies tend to shift during cut, it is suspected that the effectiveness of this approach will be limited, though no field data is yet available.

In a project related to the Kennametal tuned tooling development, a boring bar featuring an active damper for increased functionality was developed under DARPA funding by a team led by Lucent Technologies. They developed an adaptive control circuit for their internal damper, producing impressive results in a floor demonstration conducted at United Technologies Pratt\& Whitney in Hartford Connecticut. Test results indicated that the cutting conditions greatly impacted the tool performance, with some conditions favoring a damper orientation normal to the workpiece surface while others favored a tangential orientation. Thus, changing materials or cutting conditions can require removal and disassembly of the cutting head to properly orient the 
damper. This limitation is not problematic in a high production facility where a single set-up may be used to machine thousands of identical parts. But a more robust solution applicable to a wide range of cutting conditions was desired. Consequently, the Lucent team also produced an active tool holder capable of damping tool vibrations in two directions simultaneously. Good performance was demonstrated for a range of cutting conditions on different materials (Ref. 6). The only limitation of this approach is the difficulty in designing a generic interface that would enable the active clamp to be mounted to different machines. To date, plans to market this technology are still uncertain.

As an alternative to the previously mentioned vibration control schemes, the approach adopted in this project is to integrate piezoelectric actuators into the bar structure to enhance the damping through feedback control. Since no special tool holder is required, this technique provides the benefits of two axis control without the complications of machine interfacing. An added benefit of this approach is realized in actuator placement which is most effective near the tool root, far removed from the cutting zone.(Ref. 7). However, modifications of the bar near the root can adversely impact the tool's precision by reducing its static stiffness. Therefore, static stiffness reduction is an important consideration when incorporating active elements into the bar structure. From a stability standpoint, any loss of dynamic stiffness resulting from bar modifications should be easily regained through active damping.

In the first year of this two year project, we focused our efforts on three fronts: the creation of a computer simulation of the boring process for qualitative assessment of tool performance, the development and evaluation of a surrogate tool featuring four piezoelectric actuators to examine controllability and observability issues, and the application of test and analysis results to the design of a prototype bar to be manufactured and tested in the second year of the project. In the second year, a number of prototype bars were manufactured and successfully tested in the laboratory. However, a number of unanticipated delays were encountered in the cutting test phase of the project. Consequently, a complete performance evaluation was completed only for the boring bar with overhang of 6 . Although the active control showed significant vibration reduction in benchtop tests for overhangs of 8 and 10, only limiting cutting data was obtained for these cuts prior to the conclusion of the project. Nevertheless, we believe that the results obtained indicate the potential for achieving chatter free machining at these geometries.

In the following section, a boring process model is described and incorporated into a simulation within the Matlab programming environment (Ref. 8). A simplified model of the cutting action relating cutting forces to instantaneous chip area is used to drive a state space model of the boring bar (Ref. 9). This simulation provides a sufficiently realistic depiction of the machining environment for the qualitative evaluation and comparison of various boring bars. In section 3 , the development and testing of the surrogate active boring bar is then summarized. An actuation methodology suitable for controlling bending vibrations in thick bars using Lead Zirconate Titanate (PZT) stacks is summarized. A commercially available two inch diameter bar was modified to accept four piezoelectric stack actuators for controlling bending vibrations tangential and normal to the workpiece surface. Incorporating the actuators into the boring bar required significant material removal, and finite element analyses were conducted to estimate the resultant loss of static stiffness. For the cases considered, sufficient active control authority could be obtained with a less than $7 \%$ loss of static stiffness. Bench tests using a rate feedback algorithm in conjunction with a tip mounted accelerometer were used to evaluate actuator effectiveness. A favorable comparison to an unmodified bar is illustrated, with an $18 \mathrm{db}$ reduction in first mode 
amplitude achieved. Low order models were fit to the various transfer functions through application of the Eigensystem Realization Algorithm (Ref. 10) and used as input to the boring simulation. Results indicate that significant improvements in bar performance are possible through implementation the proposed active damping scheme.

In section 4, test, analysis, and simulation results form the basis for the design modifications for the prototype bar. Key issues in the design include number and location of actuators needed to establish sufficient control authority in the presence of system uncertainties. The results of the cutting tests are described in section 5. Application of active vibration control yielded significant reductions in vibration amplitudes over a range of operating conditions for a boring bar with $L / D$ of 6. Tool tip vibration time histories and power spectra provide clear evidence of the absence of chatter in the controlled cases. Furthermore, the ability of the system to halt chatter once initiated is demonstrated, with dramatic improvements in surface finish quality evident between the control on and control off cases. Finally, some closing remarks are provided in section 6. 


\subsection{Boring Process Model}

One of the primary objectives of the first year (FY96) efforts was to develop a boring process simulation to enable qualitative comparisons of the performance of various boring bars. Recognizing the complexity of the metal removal process, the intent of this effort was to create a development tool for qualitative assesment of active boring bar designs as opposed to an analysis tool that could accurately predict cutting performance for all possible cutting insert shapes and rake angles. Toward that end, only the simplest of cutting insert geometries is considered with the implicit assumption that qualitative performance evaluations based on dynamic characterizations of the boring bars are valid regardless of the cutting insert geometry. A simulation of the boring process was developed in the Matlab programming environment assuming a rectangular cutting insert with zero rake angle. Process parameters are entered through a graphical user interface, and a tool-workpiece interaction model predicts the cutting forces that drive the tool vibrations which are recorded on the workpiece surface. Under certain cutting conditions, the workpiece waviness can regenerate, leading to the development of classical chatter vibration in which the tool intermittently leaves the workpiece surface. Simulation output includes an estimate of the tool tip vibration history and the resulting workpiece surface profile.

\subsection{Dynamic Model of the Cutting Tool}

An OD (outer diameter) turning operation using a boring bar is illustrated in Figure 2.1. Because the tool workpiece interaction is similar for $\mathrm{OD}$ and $\mathrm{ID}$ (inner diameter) turning, $\mathrm{OD}$ turning is used exclusively in this program to simplify data collection and to permit the use of simpler workpiece geometries. Although the use of large tools relative to the workpiece diameter can often be justified for the case of external turning, internal boring operations impose geometric constraints on the tool selection. In all cases, the shortest fattest tool available should be used to maximize precision and tolerance to regenerative vibrations. The boring bar shown in the figure

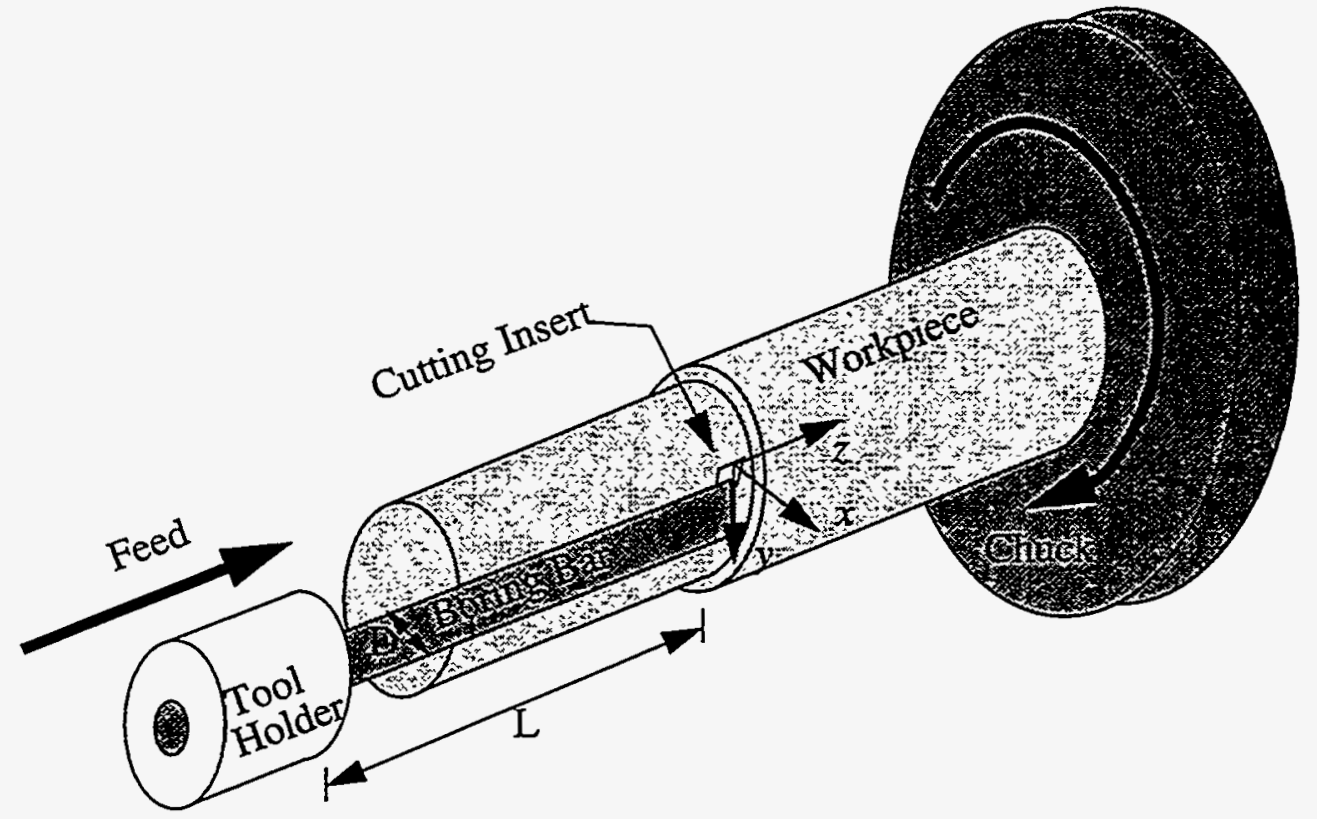

Figure 2.1. - A Depiction of External Boring Operation With Axial Infeed. 
has an effective length $L$ and diameter $D$ and is mounted to the tool holder. The bar fitted with a cutting insert travels along the surface of the workpiece with depth of cut $d$ and feed rate $f$. Throughout the entire operation, the tool and its support system move in unison, and the effective length of the boring bar remains constant.

The tool workpiece interaction yields a cutting force that excites the bending modes of the boring bar. Typically, boring bars are much stiffer in torsion than in bending and thus rotational motions of the tool are neglected. However, it is recognized that in the absence of bending vibrations, torsional modes can adversely impact the workpiece surface. It is assumed that the workpiece is rigid in comparison to the boring bar, and therefore no workpiece vibrations are considered. A radial cross section of the workpiece at the cutting location is shown in Figure 2.2. For axisymmetric bars, any two orthogonal axes located at the bar center can be taken as principal axes with no cross-coupling of the orthogonal vibrations. However, non-uniformities in the bar and the boundary conditions imposed by the chuck compromise the assumption of axial symmetry and the orientation of the principal axes is unknown. For simplicity, the $X$ and $Y$ axes are aligned in the directions normal and tangential to the workpiece surface at the cut, regardless of the orientation of the principal axes. The measured $X$ and $Y$ dynamics consequently exhibit some degree of coupling, the implications of which will be discussed in a later section.

The dynamics of the cutting process can be described by the state space model

$$
\begin{gathered}
\dot{q}(t)=A \underset{q}{q}(t)+B \underline{u}(t) \\
\underline{\sim}(t)=C \underset{q}{q}(t)
\end{gathered}
$$

in which the state vector $q(t)$ includes all the modes of interest and the output vector provides the normal and tangential tool displacements according to

$$
\underset{\sim}{y}(t)=[x(t) y(t)]^{T} .
$$

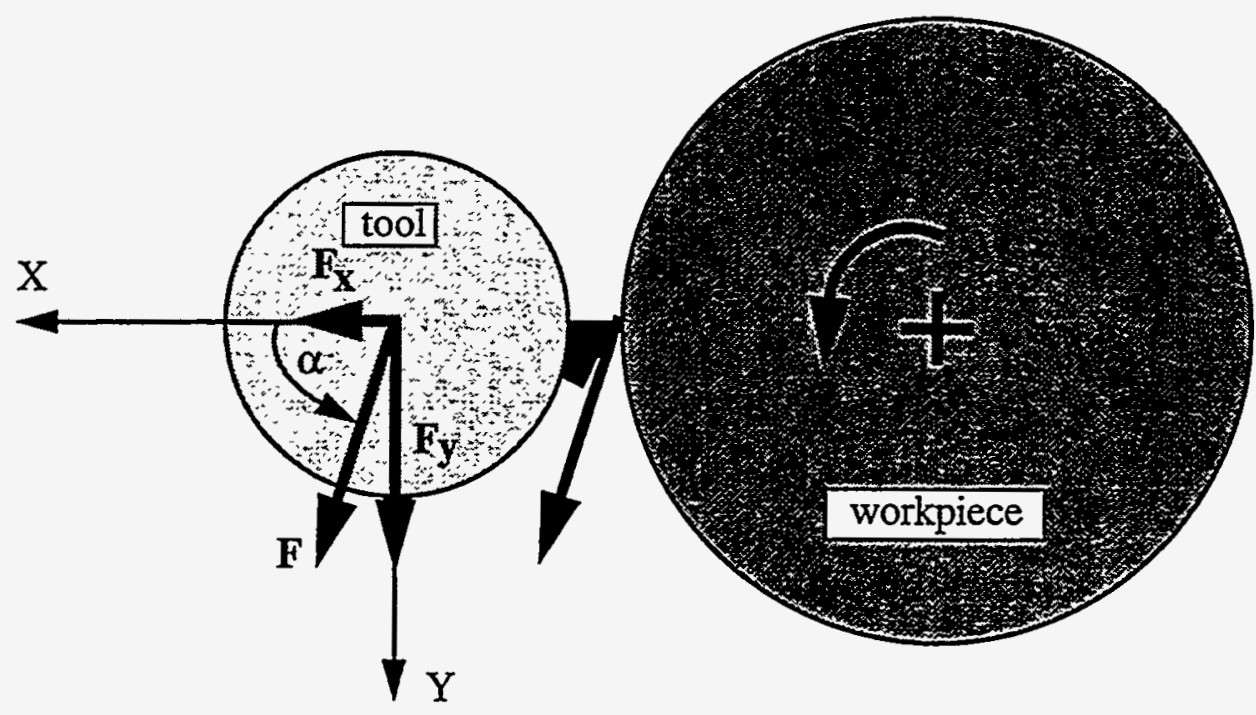

Figure 2.2. - Tool Coordinate System Defined Relative to Workpiece Interaction. 
The input vector $\underset{\sim}{u}(t)$ in equation 1 represents the normal and tangential cutting forces on the tool tip resulting from the cutting tool/workpiece interaction according to

$$
u(t)=\left[F_{x}(t) F_{y}(t)\right]^{T} .
$$

Equations 1 and 2 describe the dynamic response of the boring bar to cutting forces as seen at the tool tip. The bar dynamics represented by the $A, B$, and $C$ matrices can be obtained using analytical means, but the uncertainty in boundary conditions at the mounting chuck can result in substantial model errors. Experimentally measured frequency response functions in the normal and tangential directions at the tool tip, however, can be readily obtained and accurately reflect the boundary conditions. The other component of the interaction model, the cutting forces, are process dependent, with larger forces resulting from increased depths of cut and feed rate. A simple model for these cutting force components is described next.

\subsection{Cutting Force Model}

Although a more thorough model of the cutting process is provided by Merchant (Ref. 11), a realistic estimate is readily obtained by assuming that the force is proportional to the instantaneous area $A(t)$ of the chip being removed (Ref. 9). This approach is adopted here for its computational efficiency and because it has been shown to provide good estimates of machining stability (Ref. 12 ) in milling. The proportionality constant along the tangential direction, known as the specific cutting stiffness, has been empirically determined for a variety of materials and can be found in many machining handbooks (Ref. 13). The normal force component is related to the tangential component through the resultant force angle $\alpha$, which exhibits a dependence on a number of process parameters but is typically in the range of 70-75 degrees. Therefore, the ratio of the normal to the tangential force generally falls in the range of 0.26-0.36.

To allow for flexibility in the simulation, no dependence between the normal and tangential cutting forces is assumed. Instead, independent specific cutting stiffnesses are used to estimate the cutting forces according to

$$
\begin{aligned}
& F_{y}(t)=K_{t} A(t) \\
& F_{x}(t)=K_{n} A(t) .
\end{aligned}
$$

The cutting force model described in equations 5 and 6 relates the cutting forces to the instantaneous area of the chip being removed. Because chip area depends not only on the process parameters but also on the tool vibration history, this parameter requires closer scrutiny. Figure 2.3 shows an axial cross section of a boring operation in which a cutting insert of length $l$ is traveling along the workpiece surface with nominal depth of cut $d$ and feed per revolution $w$. Only rectangular inserts with zero rake angle are considered and the effects of tangential tool vibrations on the chip area are neglected. In the absence of tool deflections, the chip area is given by the product of the nominal cut depth and infeed. But the presence of static and dynamic tool deflections normal to the workpiece surface complicates the determination of instantaneous chip area. Accounting for the presence of some degree of cutting insert overlap between successive rotations, the chip area is given as 


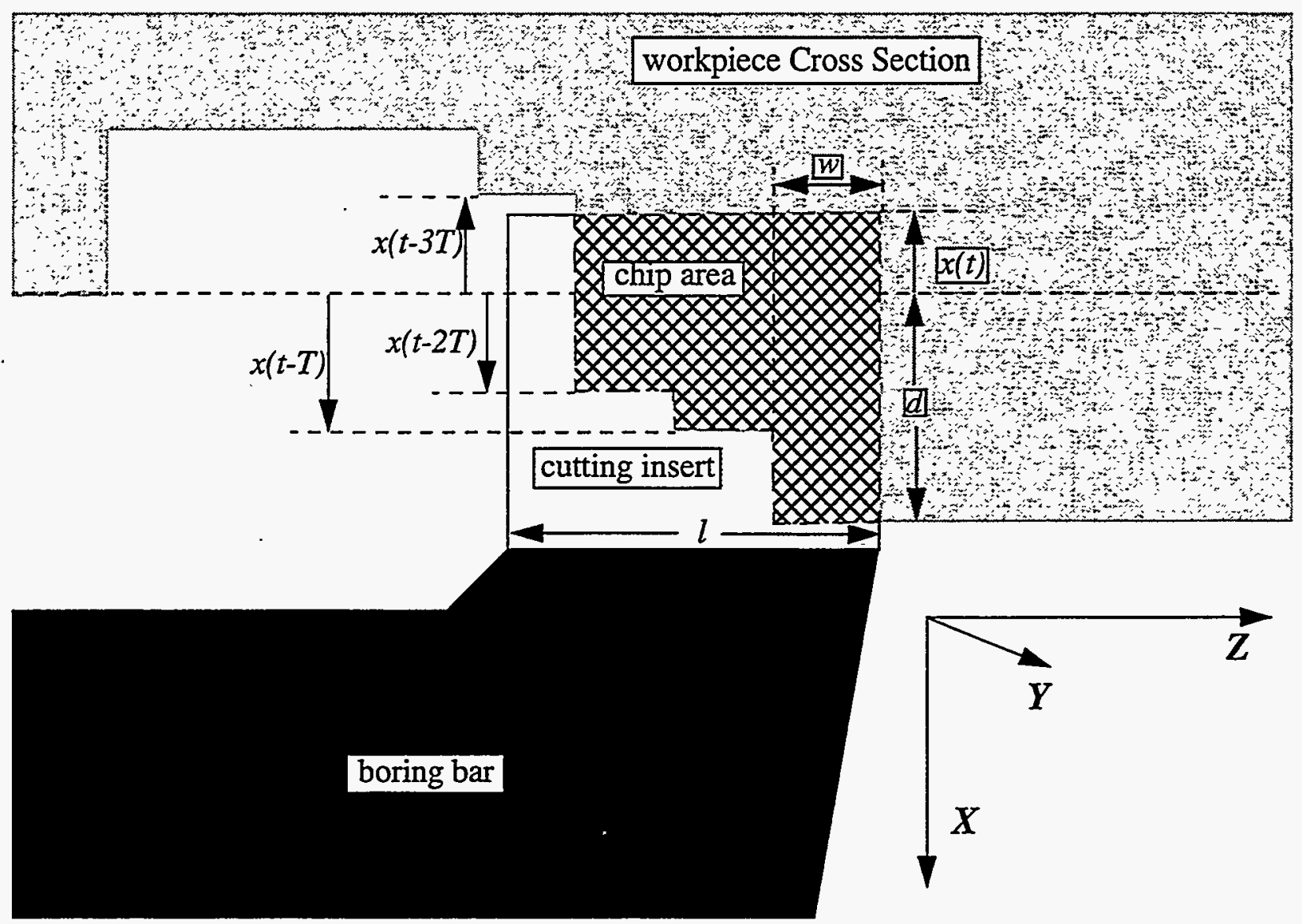

Figure 2.3. - Chip Area as a Function Process Parameters and Tool Deflection

$$
A(t)=w\{[d-x(t)]+[x(t-T)-x(t)]+\ldots+\gamma[x(t-N T)-x(t)]\}
$$

in which $T$ is the period of workpiece revolution. Each of the $N$ overlapping sections is included only if it makes a positive contribution to the chip area. For a particular overlap region, a negative contribution occurs when the tool loses contact with the workpiece, and therefore this contribution is deleted from the summation. The integer number of overlapping sections is given by

$$
N=\operatorname{ceiling}\left(\frac{l}{w}\right)-1
$$

where the Matlab function ceiling indicates rounding to the next highest integer. Accounting for the likelihood of a non-integer number of overlaps, the Nth overlap includes the fractional overlap factor $\gamma$ given by

$$
\gamma=\frac{l}{w}-\text { floor }\left(\frac{l}{w}\right)
$$


in which the Matlab function floor indicates rounding toward zero. For the operation depicted in Figure 2.3, $N=3$ and $\gamma=2 / 3$. However, at the instant shown, there is no contribution of the third overlap to the instantaneous chip thickness since $x(t-3 T)-x(t)<0$.

For stable cuts, the inherent damping in the process causes any incidental tool vibrations to dissipate, leaving a smooth surface on the workpiece. In contrast, certain cutting conditions can lead to cutting instabilities that can ruin the surface finish and damage the tool. The regenerative phenomenon of tool chatter results from the overlap of the tool onto previously cut sections. Tool vibrations are recorded on the workpiece surface during each rotation, and excite subsequent vibrations by modulating the chip area. For unstable cuts, the vibration amplitude increases with each rotation, saturating at a level that shows a strong dependence on process parameters. For simplicity, we assume in the simulation that the chatter grows until the amplitude of the vibration normal to the workpiece surface exceeds the depth of cut. The cutting force is set to zero during the portion of the oscillation in which the tool leaves the workpiece surface, and the chatter amplitude stabilizes. In practice, certain cutting conditions may yield a chatter amplitude (normal to the workpiece surface) that is considerably lower than the depth of cut. In these cases, the chatter amplitude is dictated by the tangential vibration, with saturation occurring when the speed of the vibrating tool tip exceeds the surface speed of the rotating workpiece. Thus, the tool intermittently disengages from the workpiece and the chatter amplitude stabilizes.

\subsection{Boring Simulation}

The interaction of the cutting force model with the tool dynamics has been coded into a process simulation within the Matlab programming environment (Ref. 8). The simulation package consists of three separate $\mathrm{M}$-files for controlling program input, program output, and the dynamic simulation. The program is controlled through a graphical user interface. Input parameters include nominal depth of cut, workpiece radius and speed, tool geometry, tangential and normal cutting stiffnesses, simulation duration specified in number of workpiece rotations, and a state space model representing the tool's dynamics. Graphical output includes tangential and normal tool displacement histories, workpiece waviness along the tool path, and workpiece surface profile along the axial direction. Cutting force histories are also saved and are available in the Matlab workspace.

At each step of the simulation, the rotational position of the workpiece is advanced a fraction of a revolution determined by the angular speed and the integration time step. The time step is nominally chosen to be 1/20th of the period of the fastest mode in the tool dynamics model, but it is automatically adjusted as needed to provide sufficient resolution of the workpiece circumferential profile. To calculate the cutting forces, the chip area is calculated as a function of the instantaneous tool deflection normal to the workpiece surface. Current displacement is compared to the nominal depth of cut to determine the contribution of the current rotation. In addition, the surface produced during previous rotations is compared to the current deflection, and any resulting overlap is added to the total chip area. The surface profile is then updated throughout the tool contact area, with the surface defined by the current tool deflection.

When combined with the specific cutting stiffnesses, the total chip area provides an estimate of the instantaneous cutting forces. A fourth order Runge-Kutta algorithm integrates the tool's dynamics subject to the cutting forces, and updates the normal and tangential tool displacements. 


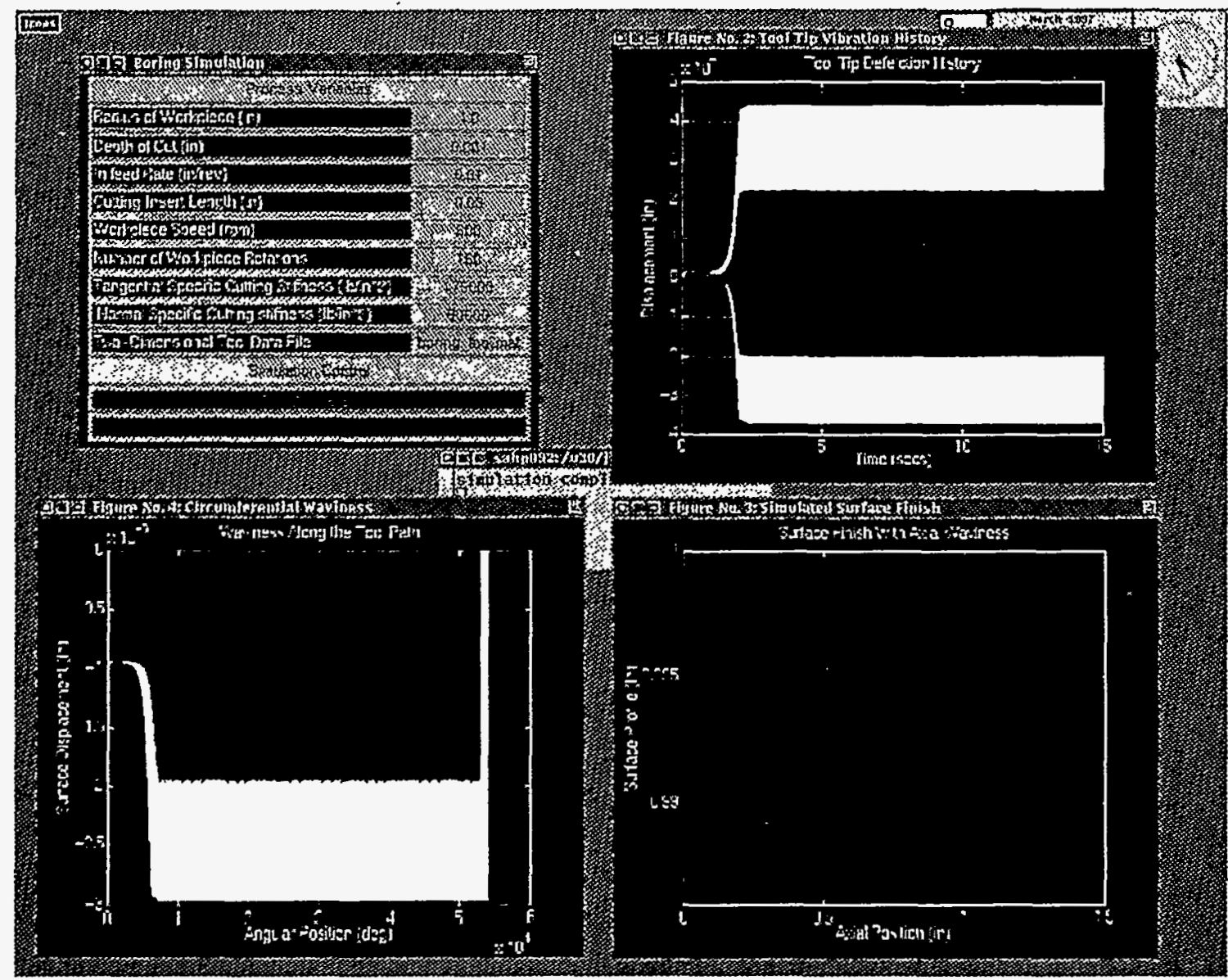

Figure 2.4. - Matlab Based Boring Simulation Tool with Input Box, Normal (magenta) and Tangential (yellow) Tool Vibration History, Tool Path Waviness, and Workpiece Profile.

Then, the workpiece rotational position is again incremented, and the cutting force estimate procedure is repeated.

A sample session is displayed in Figure 2.4, which shows an unstable cutting condition for a 2 inch diameter boring bar machining a workpiece with moderate cutting stiffness. At the start of the simulation, the tool and workpiece are not in contact. Tool immersion progresses gradually at the specified feed rate, and the static component of the cutting force slowly increases. This gradual immersion eliminates artificial transient vibrations that result from assuming full immersion with zero initial tool deflection. With the tool fully immersed in the workpiece, a static deflection of 0.0002 inches along the tangential direction is evident in Figure 2.4 prior to the initiation of chatter vibration near 2 seconds. The chatter is fully developed by 6 seconds, with a tangential and normal amplitudes of approximately 0.0043 , and 0.002 inches, respectively. A simulation of the workpiece profile is shown in the lower right portion of Figure 2.4, clearly depicting the thread-like quality of the surface that is often a consequence of chatter. 


\subsection{Surrogate Tool Development}

To aid the development of a prototype active boring bar, a surrogate tool equipped with sensors and actuators for two-axis active damping was fabricated. Four PZT stack actuators were mounted at ninety degree intervals around the root of a commercially available two inch diameter boring bar. Opposite actuators were grouped in bi-morph pairs to provide differential inputs for controlling bending vibration. Numerous benchtop experiments were conducted, and models of the open and closed loop systems were extracted for use in the boring process simulation. The purpose of this combined testing and simulation effort was to study the interaction of the active boring bar and the workpiece without the aid of expensive cutting tests. (A short series of cutting tests was reserved for the conclusion of the program to demonstrate bar performance). Results of the surrogate tool testing and analysis effort indicates that there exists sufficient control authority to significantly improve the metal cutting performance of the boring bar. The remaining issue of optimal actuator configuration will be treated in section 4 .

\subsection{Actuation Methodology}

Controlling bending vibrations in thick bars and plates requires greater control authority than is available from conventional surface mounted actuators such as PZT patches. Instead, we propose to actively damp the boring bar through the use of PZT stack actuators. Mounted in material cutouts near the bar root and offset from the neutral axis, the actuators induce bending moments in the bar through their extensional motion. To facilitate understanding of this coupling mechanism, a simple model for the actuator/bar interaction is now developed (Ref. 14).

Using a normal mode expansion, the dynamic displacement of a bar in bending is given by

$$
u(z, t)=\sum_{r=1}^{\infty} \phi_{r}(z) q_{r}(t)
$$

in which $\phi_{r}(z)$ are the mass normalized mode shapes and $q_{r}(t)$ are the modal displacements. For now the presence of actuators is not considered and the mode shapes reflect only the dynamics of the structure including any material cutouts intended to accommodate actuators. The modal control forces are the projection of the external forces onto the modal sub-space given by the inner product

$$
Q_{r}(t)=\int_{0}^{L} \phi_{r}(z) f(z, t) d z
$$

where $f(z, t)$ is the external force per unit length.

A cross section of a bar with actuators mounted opposite the beam center in cutouts is shown in Figure 3.1. The actuators contact the beam only at the end points denoted $z_{1}$ and $z_{2}$ as measured from the bar root. The effectiveness of a two-point contact as compared to a continuous bond has been described previously using shape memory alloys on thin beam structures (Ref. 15). Offset from the neutral axis by a distance $b$, the actuators have both a passive stiffness $K_{a}$ and a voltage induced strain resulting in a control force $F_{v}$. It is assumed that the actuators are mounted such that the bending stresses in the bar are not transmitted to the actuator stacks. Rather, bending 
deformation of the bar results in axial deformation of the actuators as a consequence of the offset distance.

The purpose of the actuators is to produce equal and opposite forces to control bending about the $\mathrm{Y}$ axis, reducing the lateral displacements of the beam in the $\mathrm{X}$ direction. Neglecting actuator hysteresis, the total actuator force (active and passive) is given by

$$
F_{a}(t)=K_{a}\left(p d_{33} V(t)-\Delta L(t)\right)
$$

in which $p$ is the number of wafers in the stack, $d_{33}$ is the piezoelectric constant, $V(t)$ is the input voltage, and $\Delta L(t)$ is the axial elastic deformation of the actuator resulting from bending deformations of the beam. $F_{a}$ is positive when a compressive load is present in the actuator, and the force inputs into the beam are pointing away from the actuator tips. Direct coupling between the actuator and the bar is assumed by neglecting the compliance of additional mounting hardware.

Assuming that the actuator length is small relative to the modal node spacing in the actuator vicinity, the axial actuator deformations are approximated geometrically by

$$
\Delta L_{+}(t)=b\left[\theta_{y}\left(z_{1}, t\right)-\theta_{y}\left(z_{2}, t\right)\right]
$$

and

$$
\Delta L_{-}(t)=b\left[\theta_{y}\left(z_{2}, t\right)-\theta_{y}\left(z_{1}, t\right)\right] .
$$

The subscripts + and - refer to the sign of the $X$ coordinate at each actuator's location. The beam slope $\theta_{y}(z, t)$ is obtained from the first spatial derivative of the displacement given in Equation 10 which leads to the alternate expression for the actuator deformations

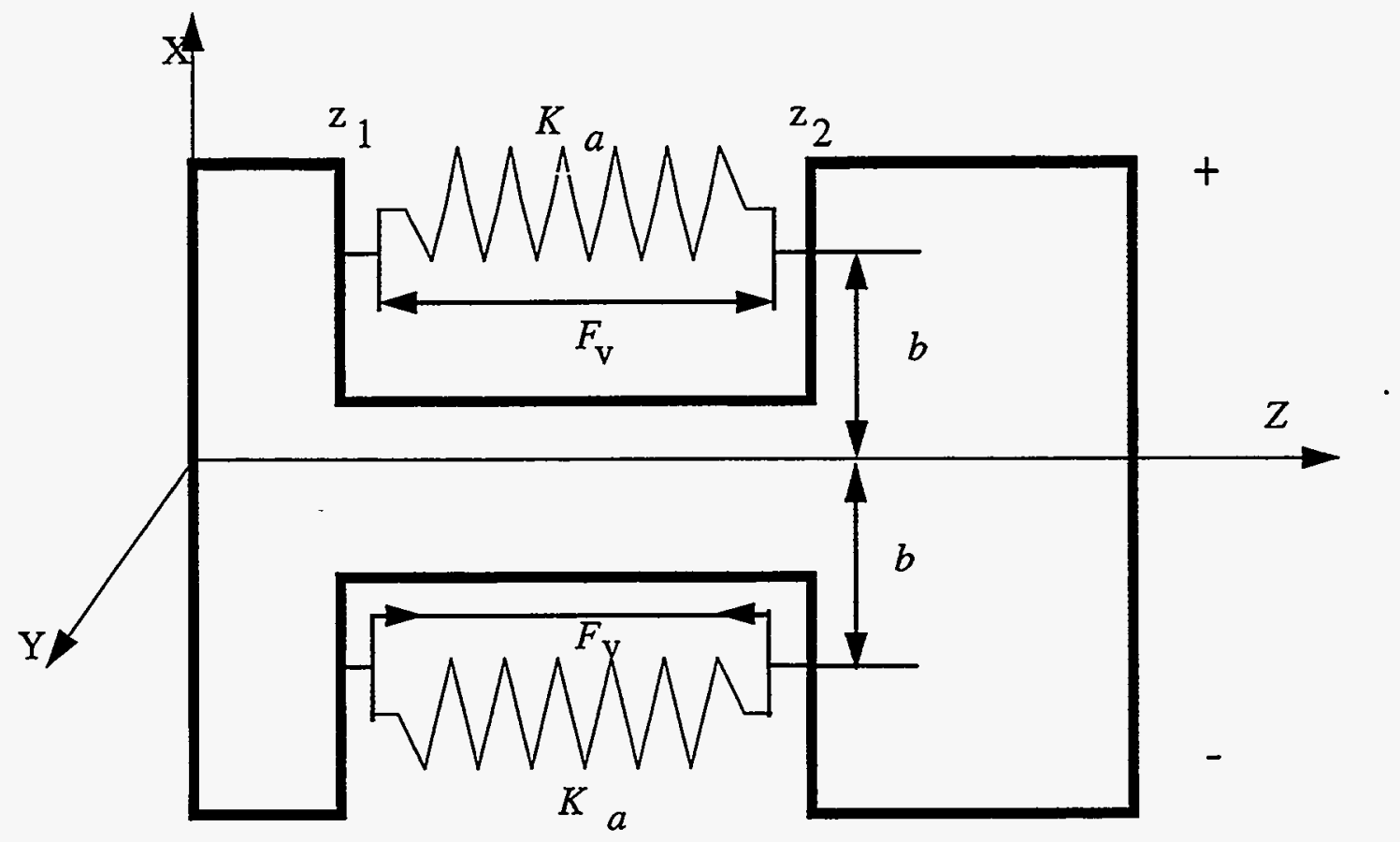

Figure 3.1. - Schematic Diagram of Actuator/Bar Interface. 


$$
\Delta L_{+}(t)=-\Delta L_{-}(t)=b\left[\sum_{s=1}^{\infty}\left(\phi_{s}^{\prime}\left(z_{1}\right)-\phi_{s}^{\prime}\left(z_{2}\right)\right) q_{s}(t)\right] .
$$

The effect of the total force (elastic and electromechanical) supplied by the actuators to the beam can be approximated as moments concentrated at the actuator-bar interfaces (Ref. 16). Since the actuators are intended to supply equal and opposite forces to the system, therefore doubling the effect of a single actuator, we make the simplifying assumption that $F_{a_{-}}(t)=-F_{a_{+}}(t)$. Then, lumping the contributions from both + and - actuators, the discrete moments at the interfaces are given by

$$
M\left(z_{1}, t\right)=-M\left(z_{2}, t\right)=2 b F_{a_{+}}(t)
$$

The moment per unit length is given in distributed form

$$
m(z, t)=2 b F_{a_{+}}(t)\left(\delta\left(z-z_{1}\right)-\delta\left(z-z_{2}\right)\right) .
$$

in which $\delta$ is the Dirac delta function indicating that the moments are zero everywhere except at the actuator interface locations. An alternate expression for the moment distribution in terms of the actuator parameters and the modal deformations is given by substituting Equations 12 and 15 into Equation 17 to yield

$$
m(z, t)=2 b K_{a}\left[p d_{33} V(t)-b \sum_{s=1}^{\infty}\left(\phi_{s}^{\prime}\left(z_{1}\right)-\phi_{s}^{\prime}\left(z_{2}\right)\right) q_{s}(t)\right]\left(\delta\left(z-z_{1}\right)-\delta\left(z-z_{2}\right)\right) .
$$

Finally, exercising the relation $f(z, t)=-\partial m(z, t) / \partial z$ (Ref. 17), substituting Equation 18 into Equation 11 and integrating by parts gives the modal control forces as

$$
Q_{r}(t)=2 b K_{a}\left[\phi_{r}^{\prime}\left(z_{1}\right)-\phi_{r}^{\prime}\left(z_{2}\right)\right]\left[p d_{33} V(t)-b \sum_{s=1}^{\infty}\left(\phi_{s}^{\prime}\left(z_{1}\right)-\phi_{s}^{\prime}\left(z_{2}\right)\right) q_{s}(t)\right]
$$

which includes both the piezoelectric effect and the elastic effect of both actuators. While the piezoelectric effect provides control authority through the applied voltage $V(t)$, the elastic component couples the bar's natural modes. An alternative derivation includes the elastic component of the actuator force in a modal expansion of Equation 10, using a different set of mode shapes and frequencies. This approach eliminates the passive coupling terms, leaving only the voltage component of Equation 12. Although it is more cumbersome, the approach presented here verifies the passive stiffening effects of the actuators.

Note that the modal control forces depend on the actuator offset distance as well as the first spatial derivative of the mode shapes. Therefore, the modal control forces can be maximized by placing the actuators in locations of peak modal strain energy and maximizing the actuator offset distance. However, as seen in Equation 15, the elastic deformations of the actuators are also directly proportional to the offset. The possibility of losing actuator pre-load as a consequence of static deflection of the tool in a cutting situation must be considered when placing the actuators. 


\subsection{Surrogate Tool Design}

To verify the feasibility of the proposed actuation methodology as a technique for enhancing the damping in the boring bar, a surrogate tool testbed was planned. Design philosophy centered on modifying a commercially available boring bar to facilitate performance evaluation through comparison with an unmodified bar. Specifically, a Kennametal model A32DDUNR4 2 inch diameter boring bar was modified to accept four Physique Instrumente Model P840.1 piezoelectric stack actuators in pockets near the bar root. The 16 inch long bar was mounted in a four jawed chuck leaving an effective tool length of 12 inches. Mounted at 90 degree intervals, the actuators function as two bi-morph actuator sets to control bending vibrations in two orthogonal axes. The pocket dimensions were nominally 2 inches in length, 0.6 inches wide, and 0.45 inches deep. Pocket depths were minimized to maintain structural integrity and to avoid interference with the bar's centrally located 0.25 inch diameter coolant tunnel. To avoid the transmission of bending stresses to the fragile PZT stacks, the actuator bar interfaces feature single point contacts with both mechanical and electrical preloads holding the actuators in place. Contact points were located at 0.5 inches and 2.5 inches as measured from the chuck jaws with a radial offset distance of 0.84 inches.

In a typical boring bar, tool length can be readily varied by sliding the tool in or out of the tool holder prior to tightening the grips. Since the actuation methodology implemented in this project is most effective when the actuators are located at the effective root of the tool near the chuck, variable length cannot be achieved using the conventional bar approach. It is envisioned that a commercial realization of the proposed active boring bar would be segmented, allowing for variable tool length by the addition or deletion of varying length 'extender' segments between the cutting tip and the root portion containing the control hardware. In this way, the actuators maintain their effectiveness by remaining at the location of highest bending strain, and the control algorithm can be readily tuned to the dynamics afforded by the new bar length.

\subsection{Static Stiffness Assessment}

Because precision in the absence of vibration is governed by the tool's static stiffness, we conducted a finite element analysis to estimate the reduction in static stiffness resulting from the bar modifications. The bar was modeled using Abaqus (Ref. 18) with 5,000 solid elements. Geometric features of the cutting tip were not modeled in detail since it was assumed that the cutting tip would have little influence on the overall bar dynamics. Spring elements were used to represent the axial stiffness of the actuators as well as the soft boundary conditions imposed by the chuck. Vendor provided actuator stiffnesses of $55 \mathrm{~N} / \mathrm{mm}$ were used in the analysis, while the boundary springs were tuned to match first mode bending frequencies taken from test data. Generic material values for steel were used to represent the bar's properties.

The results of the finite element analyses are summarized in Table 3.1, illustrating that the inclusion of the pockets reduces the static stiffness by $16 \%$. Some stiffness is recovered by the addition of the passive actuators, with the overall reduction shifting to 13.5\%. Also shown in Table 3.1 are the frequencies for the first bending modes in the $X$ (normal to workpiece) and $Y$ (tangential) directions. The bar modifications yield approximately a $3 \%$ reduction in the natural frequencies. The shapes estimated from the finite element model for these two modes are shown in Figure 3.2, in which the axial springs included to simulate the passive stiffening effects of the actuators are evident. The contours superimposed on the mode shapes reveal high strain energy 
levels in the vicinity of the actuator/bar interfaces. This result indicates that good coupling between the actuation and the critical vibrational modes can be achieved.

\subsection{Summary of Surrogate Bar Testing}

The prototype bar shown in Figure 3.3 was fabricated by Andy Jojola of department 2643, and an extensive bench top testing program was conducted. Highlights of the test program with an emphasis on the ability of the actuation approach to influence the bar damping are now summarized. Simulated performance enhancements will be summarized in the following section.

In general, the results of the surrogate tool test bed were very encouraging, suggesting the potential for achieving significant improvements in the dynamic characteristics of the boring bar. A comparison of the driving point tip transfer functions along the $X$ direction for the original bar, the modified bar without feedback control, and the modified bar using rate feedback control for active damping is shown in Figure 3.4. Although the modifications slightly compromise the static stiffness of the bar as previously described, the dynamic stiffness in the vicinity of the first mode near 205 hertz is greatly increased through the application of the feedback control, producing a 18 $\mathrm{db}$ reduction in modal amplitude. As will be illustrated in the following section, suppressing the first mode expands the performance envelope of the boring system.

\subsection{Example Simulation Based Evaluation}

To illustrate the impact active damping can have on the performance of the boring bar, dynamic models were fit to the modified bar with and without active control. These tool models

Table 3.1: Finite Element Analysis (Modified Bar Parameters Are Relative to Nominal Parameters).

\begin{tabular}{|c|c|c|c|}
\hline & Unmodified Bar & Bar With Pockets & Bar With Actuators \\
\hline \hline X Stiffness (lb/in) & 21353 & $-15.8 \%$ & $-13.3 \%$ \\
\hline Y Stiffness (lb/in) & 21868 & $-16.1 \%$ & $-13.6 \%$ \\
\hline X Frequency (Hz) & 214 & $-3.7 \%$ & $-2.8 \%$ \\
\hline Y Frequency (Hz) & 220 & $-4.1 \%$ & $-3.2 \%$ \\
\hline
\end{tabular}

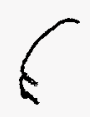
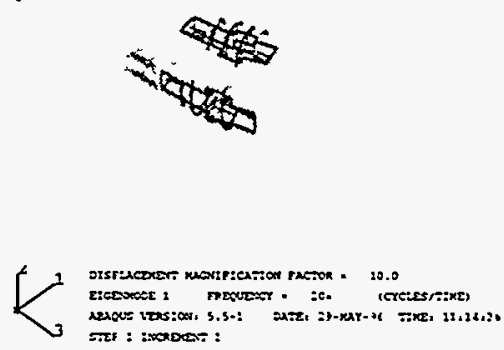

Figure 3.2. - First Two Modes for Kennametal Model A32DDUNR4 Boring Bar Including Prototype Smart Tool Modifications and Passive Actuators. 


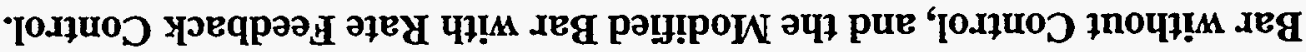

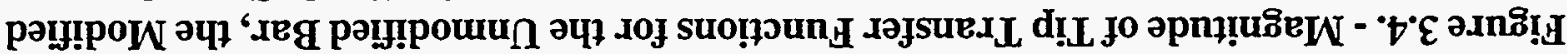

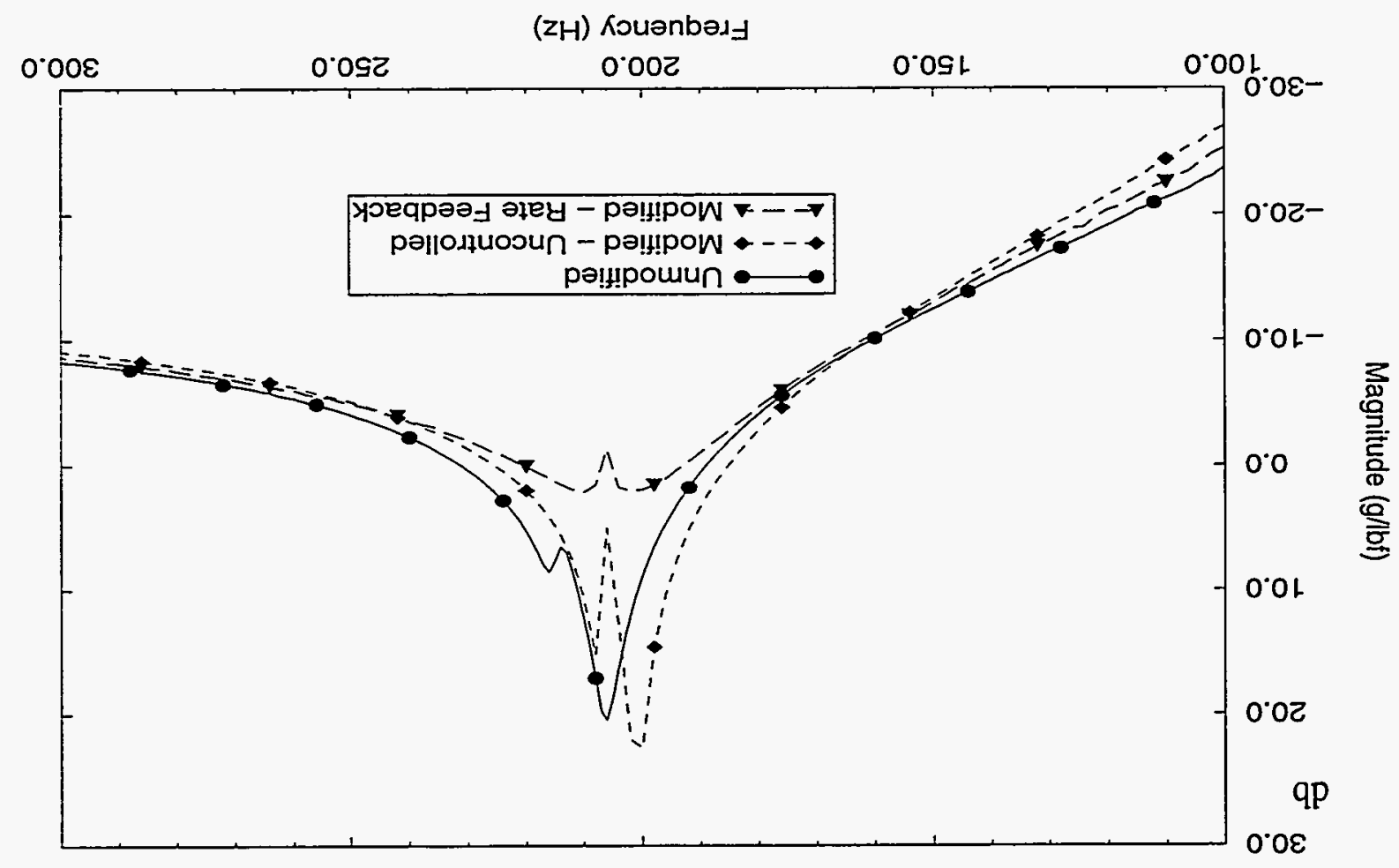

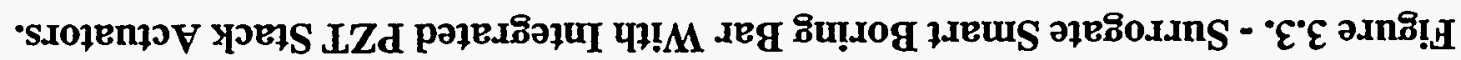

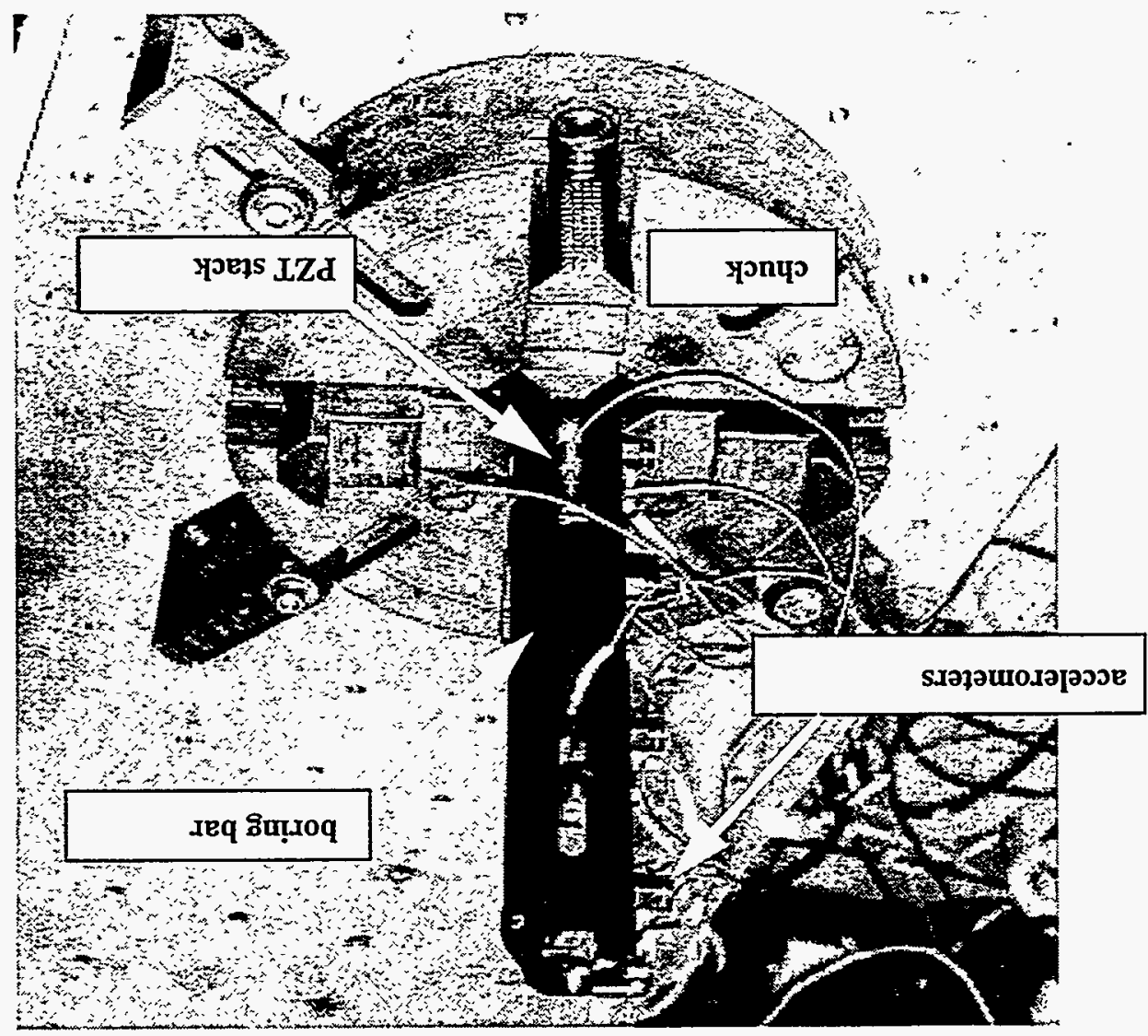


were used to simulate cutting on a 2 inch diameter cast iron bar with 0.01 inch depth of cut and workpiece speed of $600 \mathrm{rpm}$. Tool vibration history normal to the workpiece surface is shown in Figure 3.5. With an infeed rate of 0.01 inches per revolution, the uncontrolled bar produces an unstable cut, with the steady state amplitude of the chatter approximately equal to the depth of cut. The controlled case, however, shows a stable cutting condition despite increasing the infeed rate to 0.075 inches per revolution. A larger static deflection of the tool as a result of the increased cutting forces is evident in this case.

Simulated workpiece surface profiles are illustrated in Figure 3.6 which shows an axial crosssection of the workpiece near the surface. While the controlled case produces a relatively smooth surface, the surface resulting from the uncontrolled case exhibits waviness that is characteristic of chatter vibrations. In summary, the increased stability of the controlled case offers an enlarged stable machining envelope, improved surface finishes, less scrap, and longer tool life

The example presented illustrates the potential of the proposed actuation strategy for enhancing the stability of the boring bar against chatter vibrations. However, other issues related to the optimal number and placement of actuators must be considered for the prototype tool design. These issues will be further addressed in the following sections.

\subsection{Single Versus Bi-Morph Actuation}

In the previous section, the active damping results were obtained using a bi-morph actuator configuration, in which two actuators mounted on opposite sides of the bar work in unison. The benefits of this approach are two-fold. First, by supplying equal and opposite control forces (relative to the pre-load) the bi-morph configuration doubles the effectiveness of a single actuator without adding significant complexity to the control system. Secondly, the electrical and mechanical pre-loads of the actuators cancel, yielding a zero net static deflection of the bar tip in the lateral direction prior to cutting. However, this approach has substantial added cost in the form

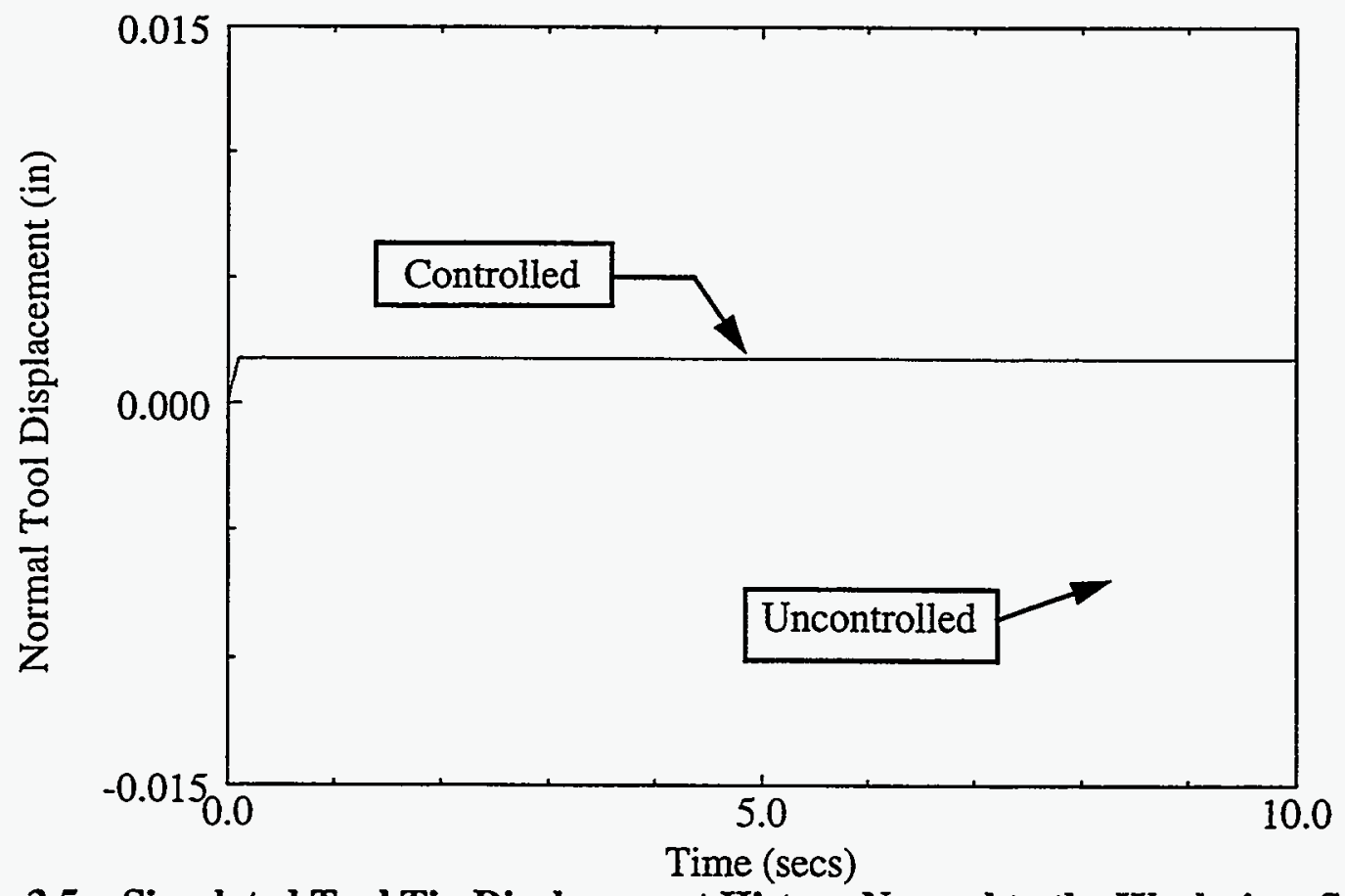

Figure 3.5. - Simulated Tool Tip Displacement History Normal to the Workpiece Surface. 
of greatly reduced static stiffness of the bar. Finite element analyses suggest that the lateral static stiffness loss resulting from inclusion of the active control hardware can be reduced to less than 7\% if only a single actuator is used. The single actuator approach is then superior if system performance is not limited by actuator force capability, and results comparable to the bi-morph case can be achieved. This appears to be the case in the surrogate bar.

Figure 3.7 shows a comparison of the $Y$ tip transfer function magnitudes of the surrogate bar for the open loop system, the closed loop system using the bi-morph actuators with a rate feedback control, and the closed loop system using one actuator in each direction with double the gain of the bi-morph case. Both normal and tangential vibration control loops were active in the two control cases which exhibited nearly identical performance. Therefore, the damping forces produced in this example were well within the capacity of the single actuator configuration. Although the redundant actuator adds a measure of robustness in the case of actuator failure, the added complexity and loss of static stiffness makes it an unattractive alternative for the prototype bar. Furthermore, static deflection resulting from a biased pre-load is not necessarily problematic, and in some cases can be beneficial. Since in all boring operations the static component of the cutting forces results in a deflection of the tool tip down and away from the workpiece, the actuator preloads can be used to partially compensate for this bias if the actuators are located on the lower and aft portions of the bar.

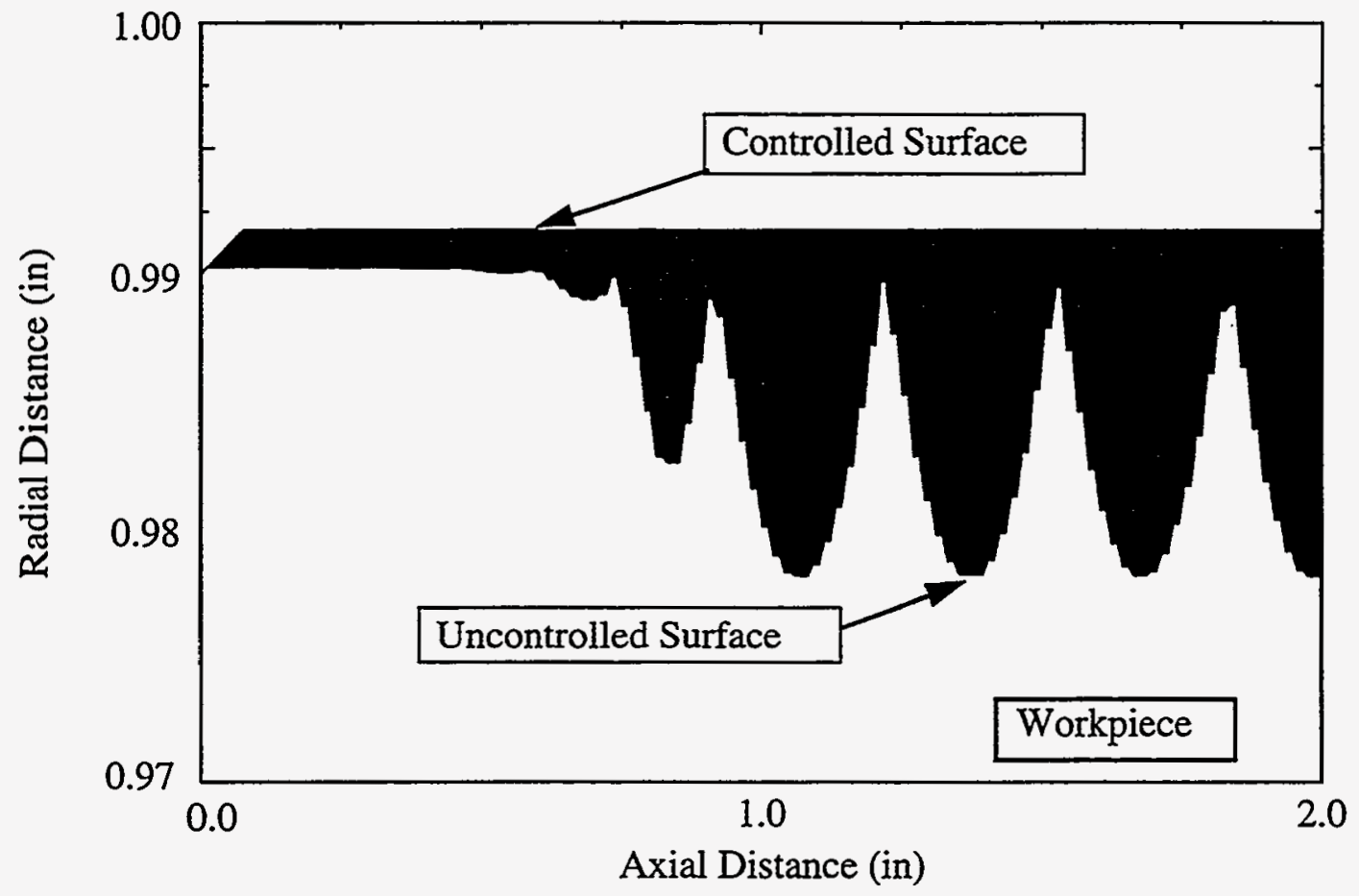

Figure 3.6. - Simulated Axial Cross-Sectional Profile of Machined Workpiece. 


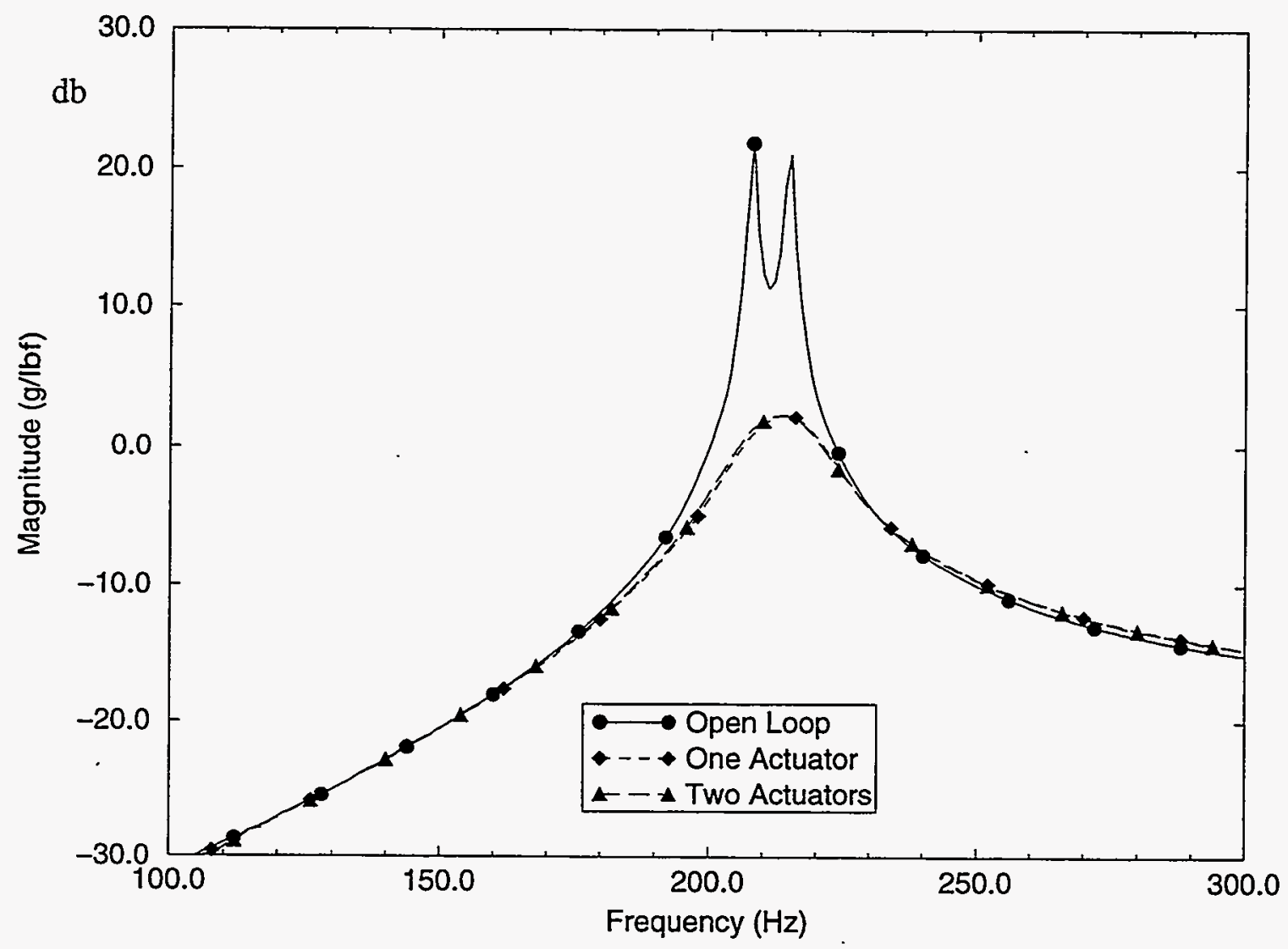

Figure 3.7. - Comparison of Y Transfer Function Magnitude With One and Two Actuators. 


\subsection{Prototype Design}

In the previous section, we developed the actuation methodology for the proposed active boring bar. Two actuator pairs were mounted at the bar root for controlling bending vibrations in the normal and tangential directions. Bench top tests combined with computer simulations showed that a single actuator could function as effectively as an actuator pair, which eliminates the static stiffness loss attributed to inclusion of the additional actuator. Furthermore, single axis actuation in the tangential direction proved to be more efficient than in the normal direction. This results from the cross-axis coupling of the bar dynamics and the higher cutting force along the tangential direction. In this section, we further explore the boring bar's stability against chatter and its dependence on principal axis orientation and actuator location. Results indicate that dual axis control is required to minimize the influence of the principal axes orientation on the cutting stability. These results formed the basis of the design for the prototype tool fabricated and tested in FY97.

\subsection{Principal Axes Orientation}

In the series of tests that was summarized in the previous section, the dynamics of the boring bar were in large part influenced by the four jawed chuck used to secure the surrogate tool to the seismic base. Minor changes in the mounting procedure altered the orientation of the bar's principal axes, affecting the amount of coupling evident in the tangential and normal directions. Since slight variations in tool mounting procedures can be expected on the shop floor, the influence of axes orientation on bar performance must be considered in order to properly place the actuators on the prototype bar.

One method of evaluation is to examine the bar's oriented transfer function which relates the bar displacement normal to the workpiece surface to the resultant cutting force. For simple turning operations, it has been shown that the limiting depth of cut is inversely proportional to the minimum real part of the oriented transfer function $G_{o r}$ written as (Ref. 19).

$$
b_{l i m}=\frac{-1}{2 k_{s}\left[\operatorname{Re}\left(G_{o r}\right)\right]_{\min }} .
$$

For cut depths below $b_{\text {lim }}$, the process is unconditionally stable, and processes that lie above $b_{\text {lim }}$ can result in unstable regenerative vibration. Thus, the minimum real part of the oriented transfer function is used here as a measure of stability against chatter vibrations, with a smaller value (in an absolute sense) indicating a more stable process.

To this end, we examine a simple two degree of freedom model of the boring bar shown in Figure 4.1. Springs and dampers are located along the principal axes of the bar, which are rotated by angle $\theta$ with respect to the normal and tangential directions denoted by $\mathrm{X}$ and $\mathrm{Y}$. The cutting force is shown inclined at angle $\alpha$ relative to the normal direction. Also present are dampers meant to represent the influence of the control actuators. The effects of these elements will be considered later and we now consider only the dynamics of the bar. In the principal coordinate system, the decoupled equations of motion are written as

$$
m \ddot{\eta}_{1}(t)+c_{1} \dot{\eta}_{1}(t)+k_{1} \eta_{1}(t)=F_{1}(t)
$$




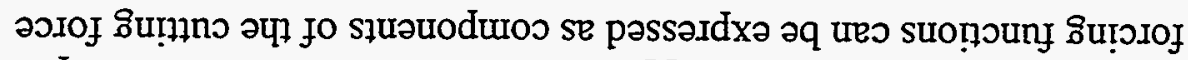

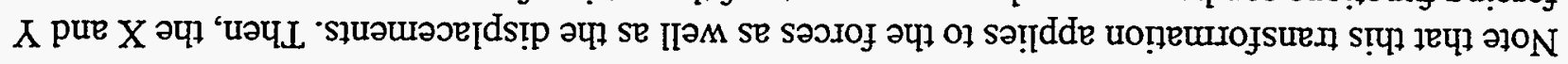

(s乙 Оᄏ)

$$
\cdot \tilde{u}_{L}=\left[\begin{array}{l}
{ }^{2} \mathrm{u} \\
I \mathrm{u}
\end{array}\right]\left[\begin{array}{cc}
\theta \text { sos } & \theta \text { u!s } \\
\theta \text { urs } & \theta \text { sos }
\end{array}\right]=\left[\begin{array}{l}
\kappa \\
x
\end{array}\right]
$$

Кq pəutjəp uo!̣ewrojsuren

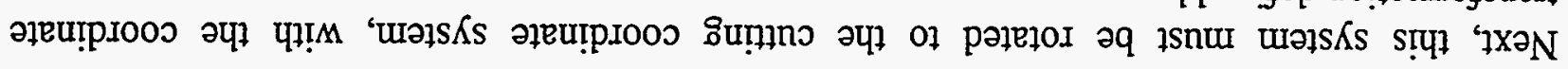
(๓乙ОВ)

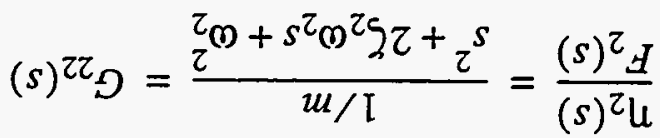

$$
\begin{aligned}
& (s)^{\mathrm{I}} D=\frac{\tau^{\mathrm{I}} \boldsymbol{l}+s^{\mathrm{I} m}{ }^{\mathrm{I}} \zeta \tau+\tau^{s}}{u / \mathrm{I}}=\frac{(s)^{\mathrm{I}} d}{(s)^{\mathrm{I}} \mathrm{u}}
\end{aligned}
$$

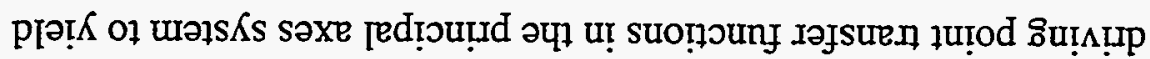

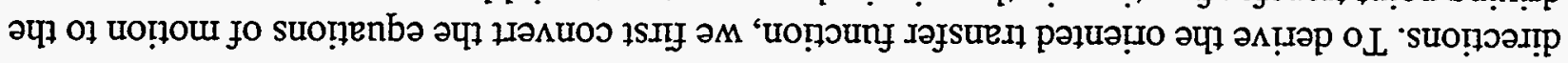

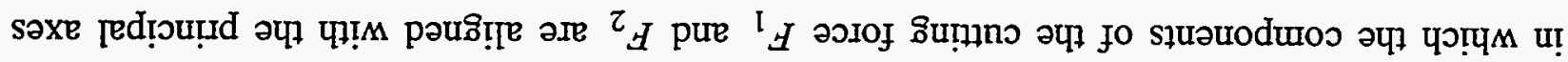
(२乙 Оᄏ)

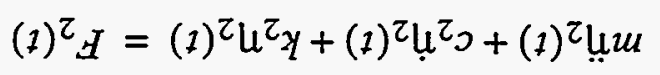

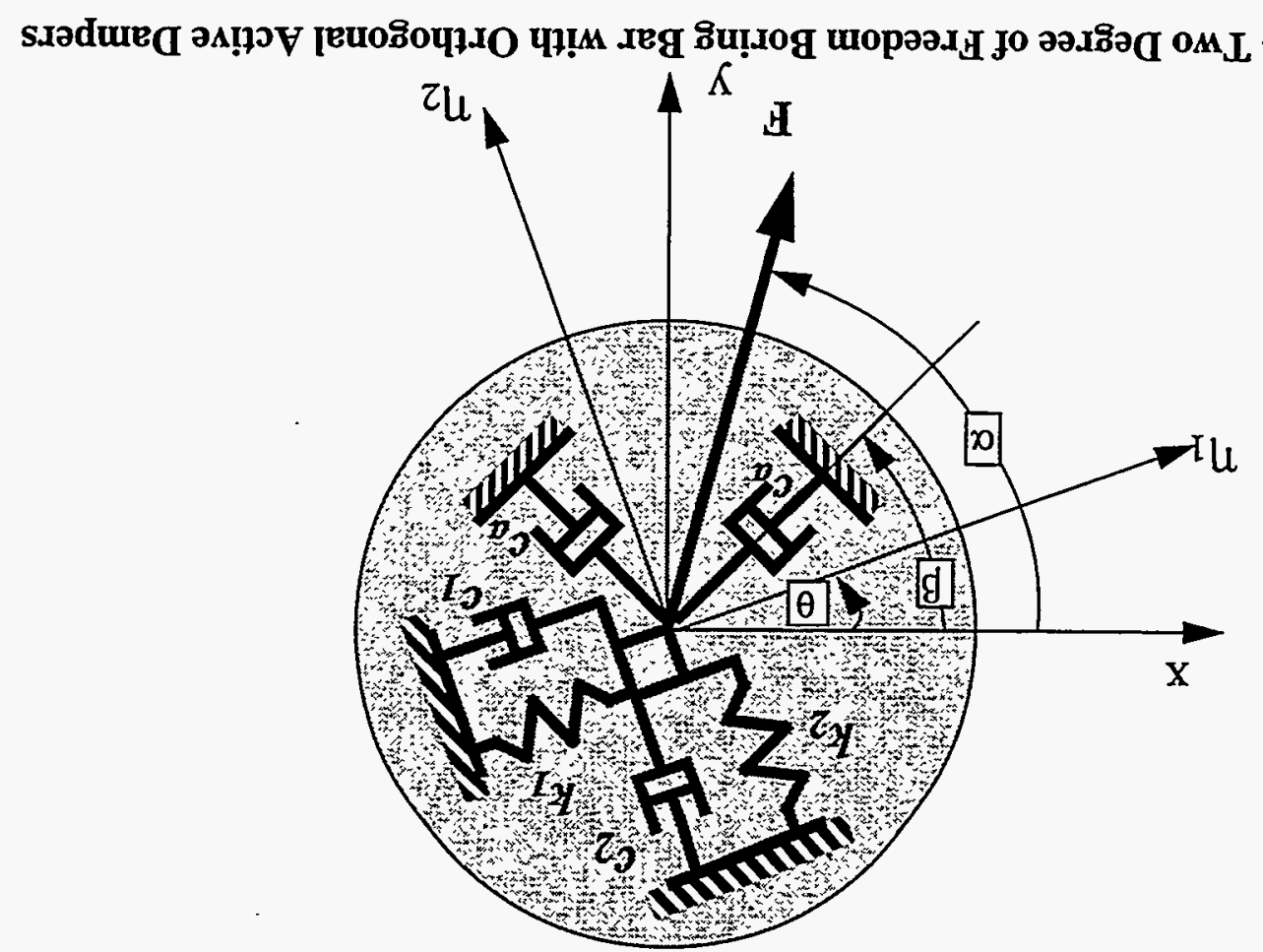




$$
\left[\begin{array}{l}
F_{x} \\
F_{y}
\end{array}\right]=\left[\begin{array}{l}
\cos \alpha \\
\sin \alpha
\end{array}\right] F .
$$

After applying the appropriate transformations, the oriented transfer function for the actuator free case is given by

$$
\frac{x(s)}{F(s)}=G_{o r}(s)=\left(\cos \theta^{2} G_{11}+\sin \theta^{2} G_{22}\right) \cos \alpha+\cos \theta \sin \theta\left(G_{11}-G_{22}\right) \sin \alpha
$$

As expected, the oriented transfer function exhibits a strong dependence on the principal axes orientation. The influence on the stability of the cutting process, however, cannot be readily deduced from examination of equation 27. Instead, a plot of the minimum real part of the oriented transfer function using the model parameters as previously described is shown in Figure 4.2. Recognizing the symmetry in the orientations, only the angles from 0 to 180 degrees are plotted. The modal frequencies $\left(\omega_{1}=214 \mathrm{hz} \omega_{2}=220 \mathrm{hz}\right)$ were taken from the finite element analysis results described in a previous section, and the lumped mass of 0.14 slugs was estimated through consideration of the estimated stiffnesses. The damping values were chosen to provide $0.5 \%$ critical damping in each mode. The cutting force angle was fixed at seventy degrees relative to the $\mathrm{X}$ axis for this and subsequent analyses. As indicated in the plot, the principal axes orientation has a surprisingly large effect on the cutting process stability despite only slight differences in the modal parameters. A cutting process with an orientation angle of 80 degrees is approximately five times more stable than a process with an angle of 35 degrees. Previous test results indicated that both outcomes are equally likely owing to subtle variations in the boring bar mounting procedure.

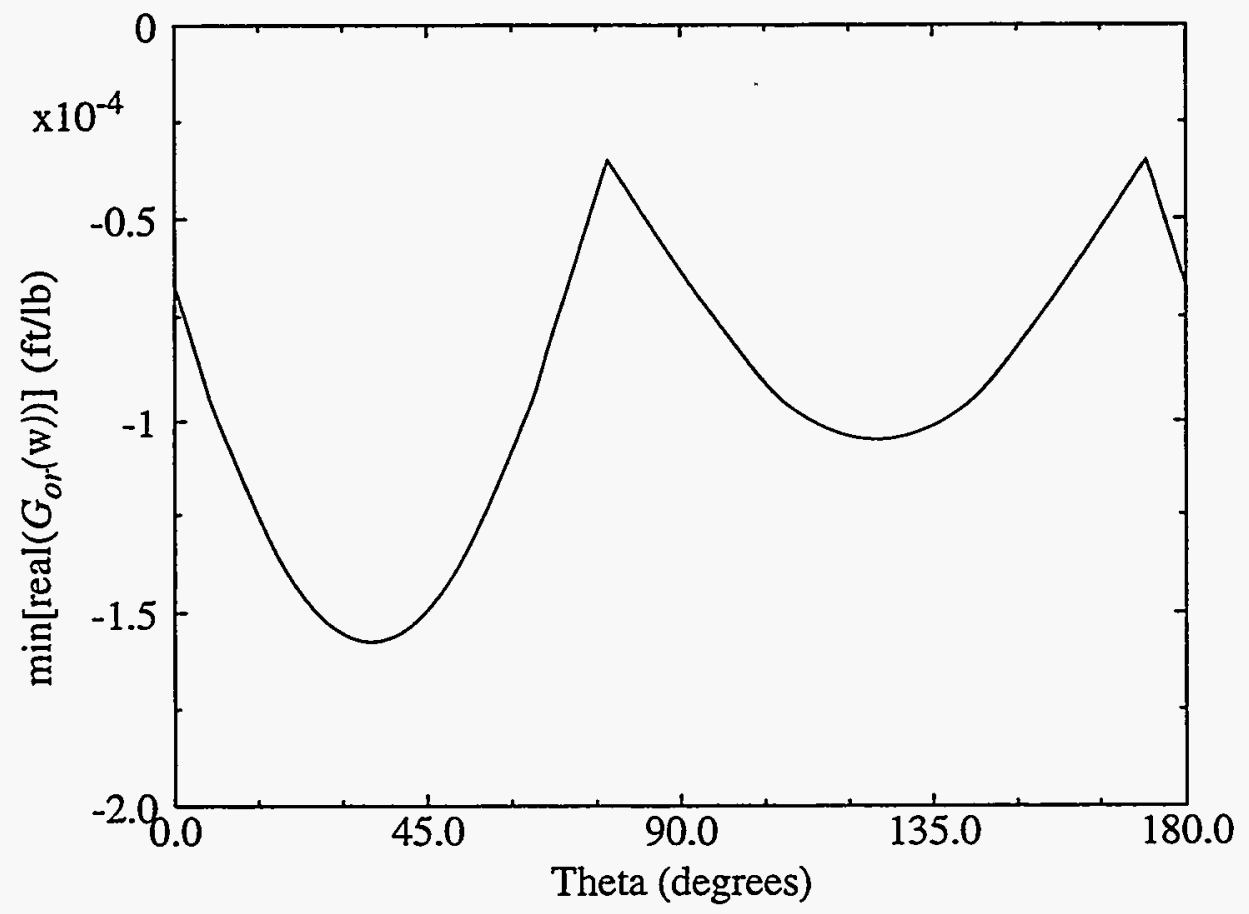

Figure 4.2. - Effect of Principal Axis Orientation on Cutting Process Stability in the Absence of Active Damping. 
Therefore, an actuator arrangement that minimizes the significance of the mounting procedure is highly desirable.

\subsection{Single Axis Control Orientation}

The main objective of this project is to effectively increase the damping of the boring bar system while minimizing static stiffness loss and system complexity. Therefore, the number and location of the actuators on the active boring bar are of primary importance. From initial studies, it was determined that the imbedded PZT actuation methodology is most effective when implemented at the root of the cantilevered bar. However, simulation results indicated that the optimal actuator configuration is not so obvious. In some cases, better performance was achieved when single axis damping was aligned with the tangential rather than the normal direction. This result is counter intuitive since it is the vibration normal to the workpiece surface that can lead to regenerative chatter. Therefore, the simple model of Figure 4.1 is now used to investigate the significance of actuator orientation.

In order to determine the optimal actuator configuration, we first consider the addition of a single actuator to the system of Equations 21 and 22. The actuator is oriented at angle $\beta$ with respect to the $\mathrm{X}$ axis, and its net effect is represented by the damping element $c_{a}$ shown in Figure 4.1. Note that an additional actuator is located orthogonal to the first, but for now only the first actuator will be considered. The modified system can now be represented as

$$
\begin{aligned}
& m \ddot{\eta}_{1}(t)+\left(c_{1}+c_{a} \cos (\beta-\theta)^{2}\right) \dot{\eta}_{1}(t)+k_{1} \eta_{1}(t)=F_{1}(t)-c_{a} \cos (\beta-\theta) \sin (\beta-\theta) \dot{\eta}_{2}(t)(E Q 28) \\
& m \ddot{\eta}_{2}(t)+\left(c_{2}+c_{a} \sin (\beta-\theta)^{2}\right) \dot{\eta}_{2}(t)+k_{2} \eta_{2}(t)=F_{2}(t)-c_{a} \cos (\beta-\theta) \sin (\beta-\theta) \dot{\eta}_{1}(t) \text { (EQ 29) }
\end{aligned}
$$

which illustrates the resulting cross coupling of what were the principal axes. A transformation of coordinates similar to that previously described can then be undertaken to arrive at the oriented transfer function.

For this analysis, the actuator damping is fixed such that it provides $5 \%$ critical damping along its axes, which is a conservative value based on the results of the experiments performed on the surrogate bar. The minimum real part of the oriented transfer function is plotted in Figure 4.3 for the range of principal axes orientations and actuator locations. As in the previous case, symmetry permits examination of the 0 to 180 degree range for both variables. For many axes orientations, having the actuator aligned with the $X$ axis $(\beta=0)$ provides a near optimal result in that it minimizes (in an absolute sense) the real part of the oriented transfer function. But this is not valid for all axes orientations. In particular, for the case of $\alpha=60$ degrees, an actuator inclined 60 degrees to the $\mathrm{X}$ axis provides better than a two-fold increase in stability as compared to an actuator aligned with the $X$ axis. In addition, as compared to axes orientations in the 100 to 180 degree range, the damper is relatively ineffective for orientations ranging from 30 to 50 degrees.

As previously noted, the mounting procedure can have a dramatic impact on the system dynamics, making virtually any orientation of the principal axes possible. The analysis summarized in Figure 4.3 indicates that satisfactory performance cannot be guaranteed using a single actuator. For some orientations, an actuator aligned normal to the workpiece will provide the best possible result, while in other cases cutting stability can be maximized using a tangentially 
aligned actuator. The possibility of achieving maximum performance through the inclusion of a second actuator is now considered.

\subsection{Dual Axis Control Orientation}

In an effort to create an active boring bar design that minimizes the influence of axes orientation on closed loop performance, we now consider the addition of a second actuator mounted orthogonal to the original as shown in Figure 4.1. Assuming that the damping provided by each actuator is identical, the equations of motion in the principal axes coordinate system are given by

$$
\begin{aligned}
& m \ddot{\eta}_{1}(t)+\left(c_{1}+c_{a}\right) \dot{\eta}_{1}(t)+k_{1} \eta_{1}(t)=F_{1}(t) \\
& m \ddot{\eta}_{2}(t)+\left(c_{2}+c_{a}\right) \dot{\eta}_{2}(t)+k_{2} \eta_{2}(t)=F_{2}(t) .
\end{aligned}
$$

Thus, the addition of the second actuator orthogonal to the first eliminates the dependence of the dynamics on the actuator locations. After again transforming coordinates to arrive at the oriented transfer function, the performance of the boring bar is summarized in Figure 4.4. Note that although the minimum real part of the oriented transfer function again varies over the range of principal axes orientations, the worst case scenario for the two actuator case $(\theta=35$ degrees) offers a ten fold improvement over the worst case expected for the single actuator case. In addition, the best and worse performance for the two actuator case differ by less than a factor of two as compared to greater than ten for the single actuator case.

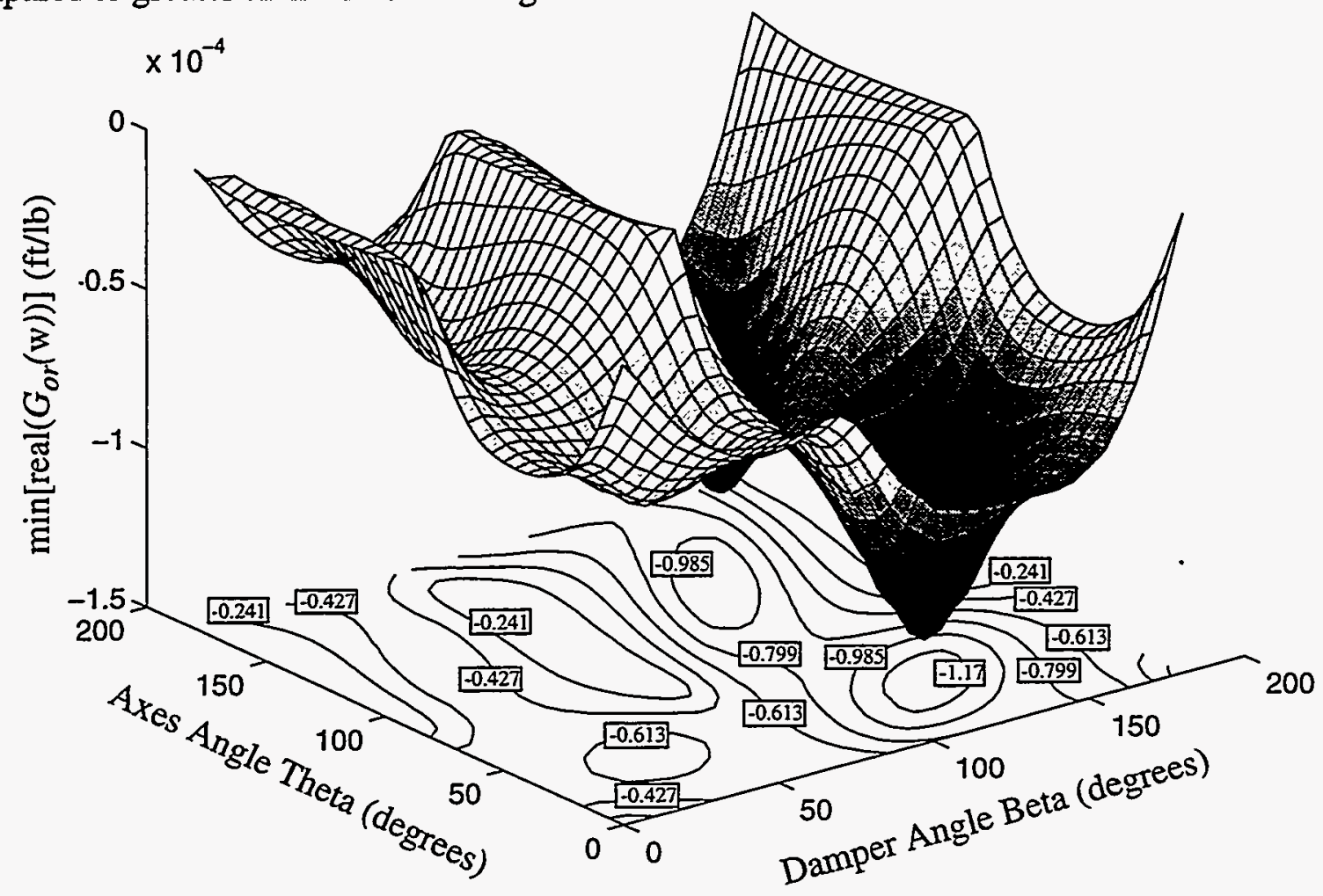

Figure 4.3. - Cutting Process Stability As A Function of Axes Orientation and Actuator Location. 


\subsection{Prototype Design Recommendations}

The analysis presented in this section coupled with the surrogate tool test results summarized in the previous section form the basis for the prototype active bar design. Single as opposed to bimorph actuator sets were to be used since this configuration was shown to provide adequate control authority while minimizing the boring bar's static stiffness loss resulting from actuator integration. Two orthogonal actuators were to be mounted in the normal and tangential directions on the lower and aft sides of the bar relative to the workpiece. Although any orientations would suffice, these directions were selected because the static deflection of the boring bar subject to the cutting forces will result in compressive loads at the actuator locations. This eliminates the possibility of pre-load loss due to static deflection that would exist for actuators mounted opposite the chosen directions. Furthermore, the mechanical and electrical pre-load of the actuators at the chosen locations have the added benefit of compensating for the tool's static deflection which leads to dimensional errors on the finished workpieces.

Valenite brand boring bars in the two-inch diameter class were selected as the host boring bars for prototype development. A pristine boring bar of identical make and model was left unmodified to enable quantitative assessments of the active boring bar performance. Physik Instrumente PZT stack actuators were mounted in cutouts at the bar root for vibration control. A self sensing actuator scheme in which the selected piezoelectric stacks function simultaneously as both a sensor and an actuator was carefully considered for this application (Ref. 20). This approach is attractive in that collocated sensors and actuators enable the use of low order control algorithms with stabilizing spillover into the uncontrolled modes. However, it was determined that tip mounted accelerometers were more suited as sensors for this vibration control task due to the low strain

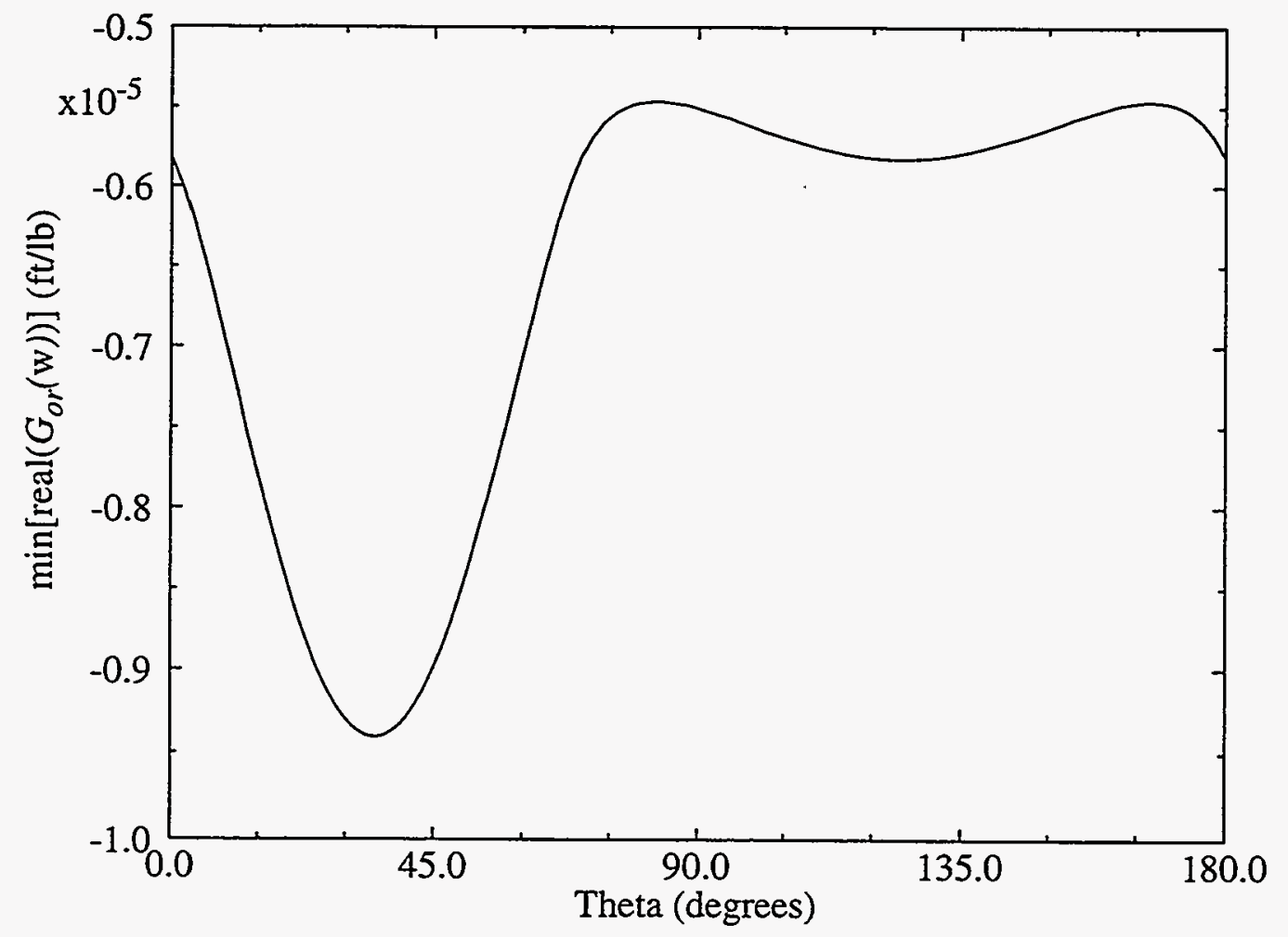

Figure 4.4. - Cutting Process Stability as a Function of Axes Orientation with Two Orthogonal Active Dampers. 
levels registered at the actuator locations. Although a number of feedback control schemes were considered on the surrogate tool, rate feedback produced damping levels comparable to more sophisticated algorithms. Thus, this approach was chosen over other techniques for the prototype bar because of its simplistic implementation. 


\subsection{Cutting Test Summary}

In the second year of this two year effort, project emphasis was placed on applying the knowledge obtained during the first year toward the development and testing of the prototype boring bars. Toward that end, a number of two inch diameter boring bars of varying lengths were purchased from Valenite and modified to incorporate the active control hardware. After completing a benchtop characterization program, we embarked on a series of cutting tests aimed at quantifying the performance enhancements obtained through active vibration control for bars with overhangs of 6,8, and 10. Excellent results were obtained for the overhang 6 bar and are summarized in this section. As of this writing, available test data from the bars with overhangs of 8 and 10 is yet inconclusive. Unfortunately a number of unanticipated factors combined to limit our machine time, and we were unable to complete a thorough survey for these test articles. However, bench-top testing of these bars indicates that the active vibration control (AVC) provides damping comparable to that for overhang of 6 . Thus, it is believed that performance levels comparable to that described in this section can be achieved for overhangs of 8 and 10 .

\subsection{Test Program Overview}

Because initial test efforts were hampered by the unpredictable nature of the host machine tool's dynamics, a survey of the various turning machines housed in building 840 was undertaken to find a suitable machine for the cutting tests. At the conclusion of this effort, the Binns and Barry horizontal lathe (machine 93A) shown in Figure 5.1 was selected on the basis of its rigid tool

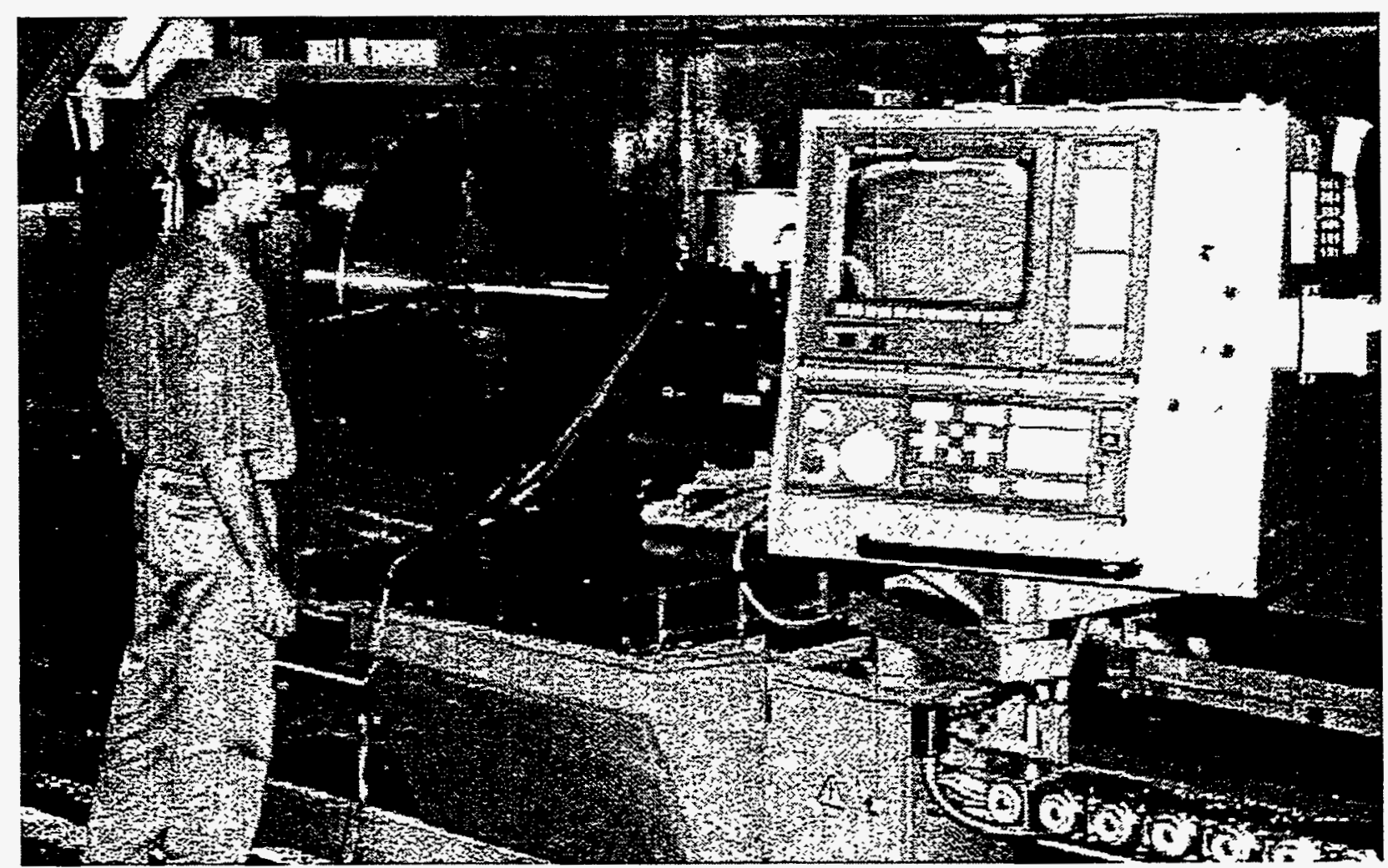

Figure 5.1 - Binns and Barry Horizontal Lathe (machine 93 A) Housed in Building 840 at Sandia Albuquerque. 
holding system and its repeatable dynamics. All tests reported here were conducted under the supervision of machine operator Terry Litts (Department 1481).

The prototype boring tool shown during a cut in Figure 5.2 consisted of a two inch diameter steel shank Valenite bar (model S32-MCLNL6) fitted with a titanium nitride coated cutting Valenite cutting insert (model CNMP643 SV4). All cutting tests described in this report were performed using an 6 inch diameter 4340 steel workpiece that had been heat treated to a hardness of 42 Rockwell ' $\mathrm{C}$ ' scale. The bar was modified to incorporate Physik Instrumente PZT stack actuators (model 840.1) near the bar root as shown in Figure 5.3. The actuators were mounted using mechanical preload, with the rounded actuator fittings being housed in the concave pocket ends. Based on the studies completed in FY96, only two actuators were used to minimize the static stiffness loss resulting from the bar modifications. The actuators were mounted on the lower and aft sections of the bar to suppress bending vibrations tangential and normal to the workpiece surface, respectively. Tip mounted Endevco accelerometers (model 22) provided the input to the two independent rate feedback control algorithms running on the DSpace controller. System condition was monitored using an Hewlett Packard 3565s system operating at 4096 samples per second.

The test series completed on the Binns and Barry lathe consisted of open and closed loop frequency response functions of the tool mounted in the tool holder, open and closed loop point cutting comparisons, and a cutting survey to compare the overall performance of the prototype tool with an unmodified bar of the same make. Each of these efforts is now briefly described.

\subsection{Frequency Response Functions}

A comparison of the normal and tangential frequency response functions is given in Figure 5.4 and 5.5, respectively. A double pole is evident in the vicinity of $330 \mathrm{~Hz}$, indicating the presence of the first bending mode in both the normal and tangential directions. Note that the resonant frequency of the bar is considerably higher than that of the surrogate bar, indicating that the machine's tool clamp is much more rigid than the chuck used in the bench top tests. A $15 \mathrm{db}$ reduction in the resonant peaks result from the application of AVC. While this reduction is considerably less than the $18 \mathrm{db}$ drop achieved in the benchtop tests, the difference can be attributed to a higher initial damping in the machine mounted tool which effectively reduces the impact of the actuator inputs. For this machine mount case, the tangential and normal modal damping ratios increased from 2.1 and 1.5 percent to 9.0 and $4.0 \%$ respectively. Thus, a significant

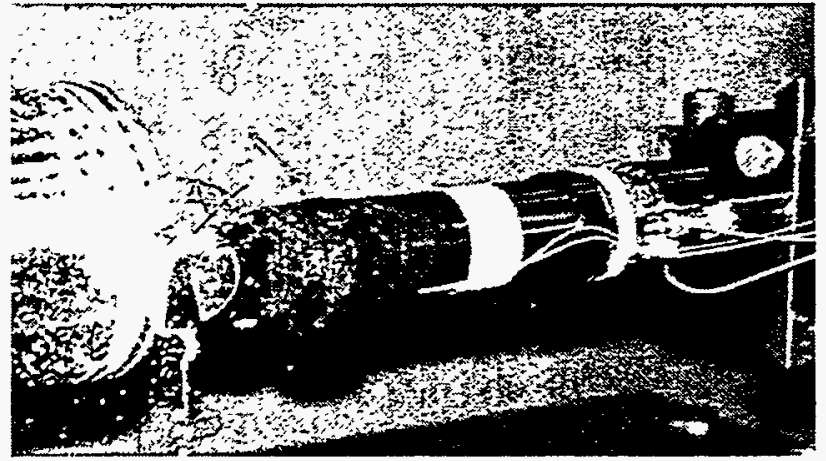

Figure 5.2 - Prototype Valenite Boring Bar with $L / D$ of 6.

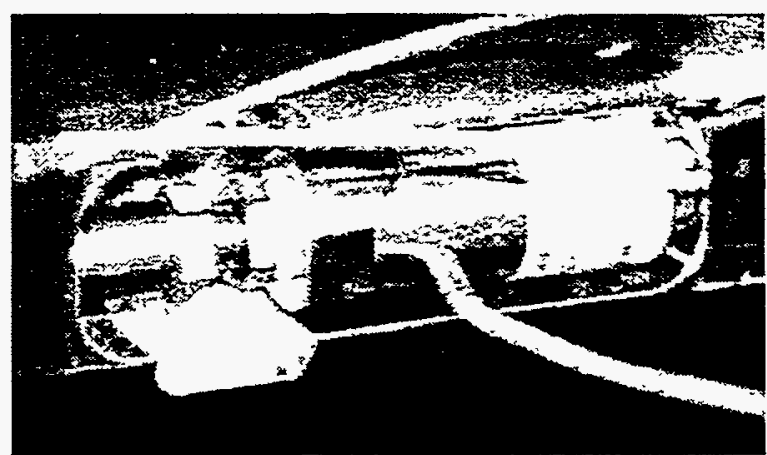

Figure 5.3 - Physik Instrumente Actuator Mounted in Cutout Near the Bar Root. 


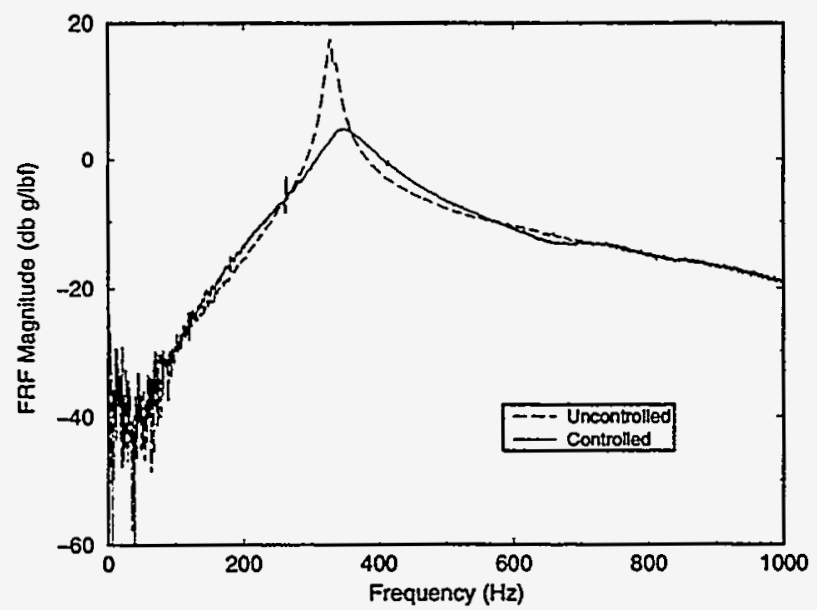

Figure 5.4 - Tangential Tip Driving Point

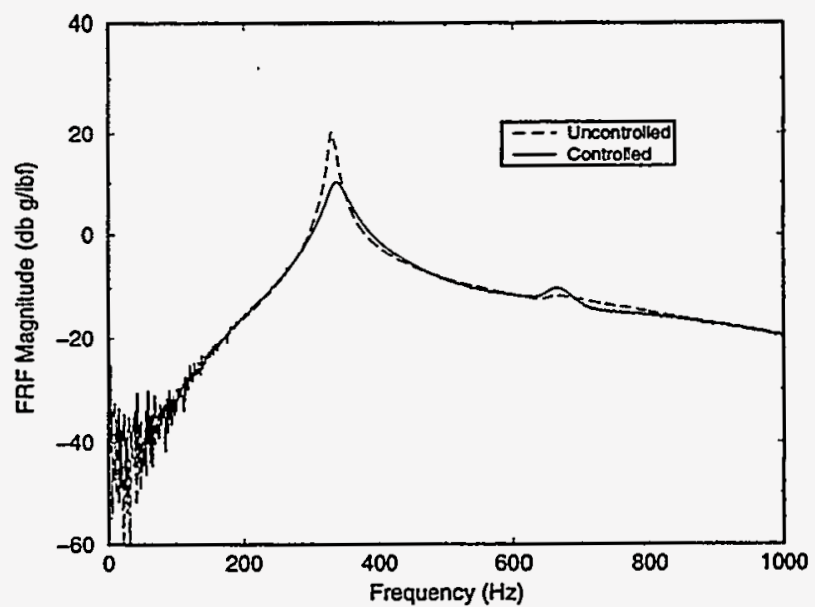

Figure 5.5 - Normal Tip Driving Point FRF

FRF Magnitude for $L / D=6$ on Machine 93A. Magnitude for $L / D=6$ on Machine 93A. improvement in cutting performance between the open and closed-loop cases can still be expected for the modified bar as shown in section 5.3. However, a truer measure of performance enhancement is obtained by comparing the AVC bar to an unmodified bar of similar make. Such a comparison is made in section 5.4.

\subsection{Cutting Demonstrations}

In this section, four different sets of cutting conditions are examined to illustrate the difference in bar vibration between the control-on and control-off cases. In the absence of AVC, the data clearly indicates the existence of chatter for each case considered. Chatter is eliminated with the activation of the AVC, but varying degrees of ambient vibration persist depending on the cutting conditions. In general, the higher depths of cut led to higher vibration levels. Note that although these examples provide insight into how the AVC influences behavior, they do not adequately reflect the potential of the AVC. For some cutting conditions, AVC may be unable to eliminate chatter once its initiated because the stroke capacity of the actuators are exceeded. However, under the same cutting conditions, the presence of AVC from the start of the cut may prevent chatter from developing at all. Therfore, the successful chatter prevention envelope under AVC is actually much larger than the chatter elimination envelope.

Although vibration normal to the workpiece surface is the driving force behind the development of chatter, controlling the tangential vibration proved to be critical to the elimination of chatter as a consequence of its larger vibration amplitude and the tangential-normal vibration coupling previously described. In addition, the tangential vibration played an important role in defining the characteristics of the chatter vibration when it occurred. Contrary to the assumption made in section 2, the chatter amplitude normal to the workpiece surface was significantly less than the depth of cut in each case considered. Instead, the saturation amplitude revealed a dependence on the speed of the workpiece, indicating that the chatter grew until the cutting tip intermittently separated from the workpiece as a consequence of the tangential vibration. This occurred when the speed of the vibrating tool tip along the tangential direction exceeded the surface speed of the workpiece. This phenomenon will be further examined in future efforts to develop an analytical model for predicting chatter amplitude as a function of machining parameters. 


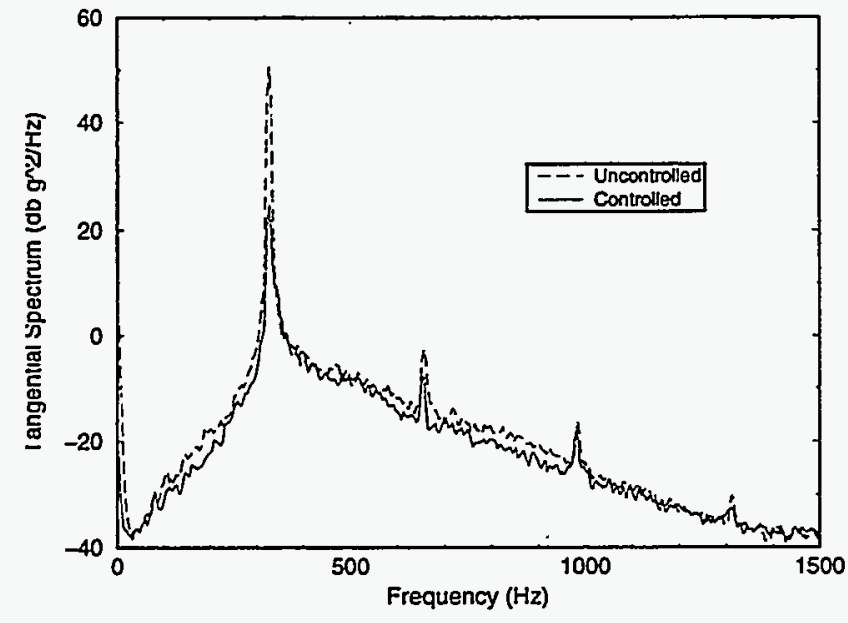

Figure 5.6. - Tangential Acceleration Spectrum for 5 mil Depth of Cut.

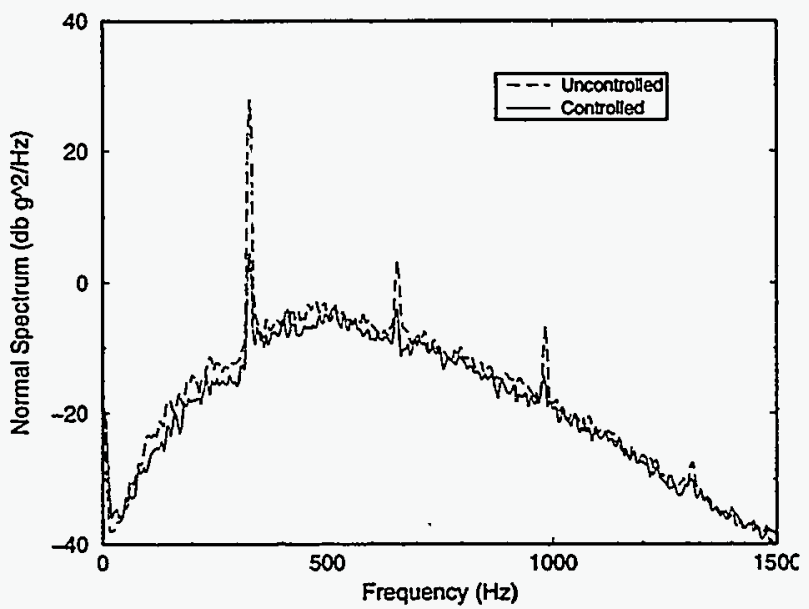

Figure 5.7. - Normal Acceleration Spectrum for 5 mil Depth of Cut.

\subsubsection{Depth of Cut 0.005 inches}

For this demonstration, the workpiece speed was set to $112 \mathrm{rpm}$ and the feed was set to 0.006 inches/rev. With the control on and off, the tangential and normal tool tip accelerations were monitored for 10 second intervals. With AVC off, the power spectra shown in Figures 5.6 and 5.7 indicate that heavy vibration was sustained near the resonant frequency of $330 \mathrm{hz}$. AVC reduced the vibrational energy by two orders of magnitude, although a strong resonant vibration persisted. The tool tip displacements were estimated through a broad band frequency domain integration of the acceleration signals. The resulting tangential-normal displacement portrait given in Figure 5.8 reveals the dominant nature of the tangential vibration, with an amplitude 10 times greater than the normal vibration. Thus, suppressing the tangential vibration was critical to the success of the AVC. Apparent in all test cases, the uniformity of the displacement portrait is indicative of the synchronous motion that is a consequence of the coupled normal and tangential vibrations. For this example, AVC produced a marginal improvement in surface finish from 83 to $53 \mu$ inches.

\subsubsection{Depth of Cut 0.010 inches}

With the cut depth set at 0.010 inches and the AVC off, the cutting speed and feed rate were adjusted until a heavy chatter was sustained at $127 \mathrm{rpm}$ and 0.0045 inches/rev. Acceleration measurements were recorded for 10 seconds prior to activating the AVC, after which 10 more seconds of data were recorded. Power spectra for these conditions are shown in Figures 5.9 and 5.10. While the existence of the strong resonant peak and several harmonics for the uncontrolled case give strong evidence thar the bar was chattering, the elimination of the peaks with the AVC on indicate that the chatter was completely eliminated. The vibrational energy at the resonant peak for this case was reduced by nearly $60 \mathrm{db}$. Consequently the estimated displacement portrait given in Figure 5.11 shows a that the tool tip displacements were dramatically reduced. Overall, the surface finish shown in Figure 5.12 improved from $140 \mu$ inches for the uncontrolled case to 54 $\mu$ inches in the presence of AVC.

The transition between chatter and no chatter machining offers interesting insight into the interaction of the control with the nonlinear metal cutting process. To illustrate, a 10 second clip of the tangential acceleration history is shown in Figure 5.13. Heavy chatter is evident in the data prior to activation of the control system at approximately 1.5 seconds. After activation, a short 


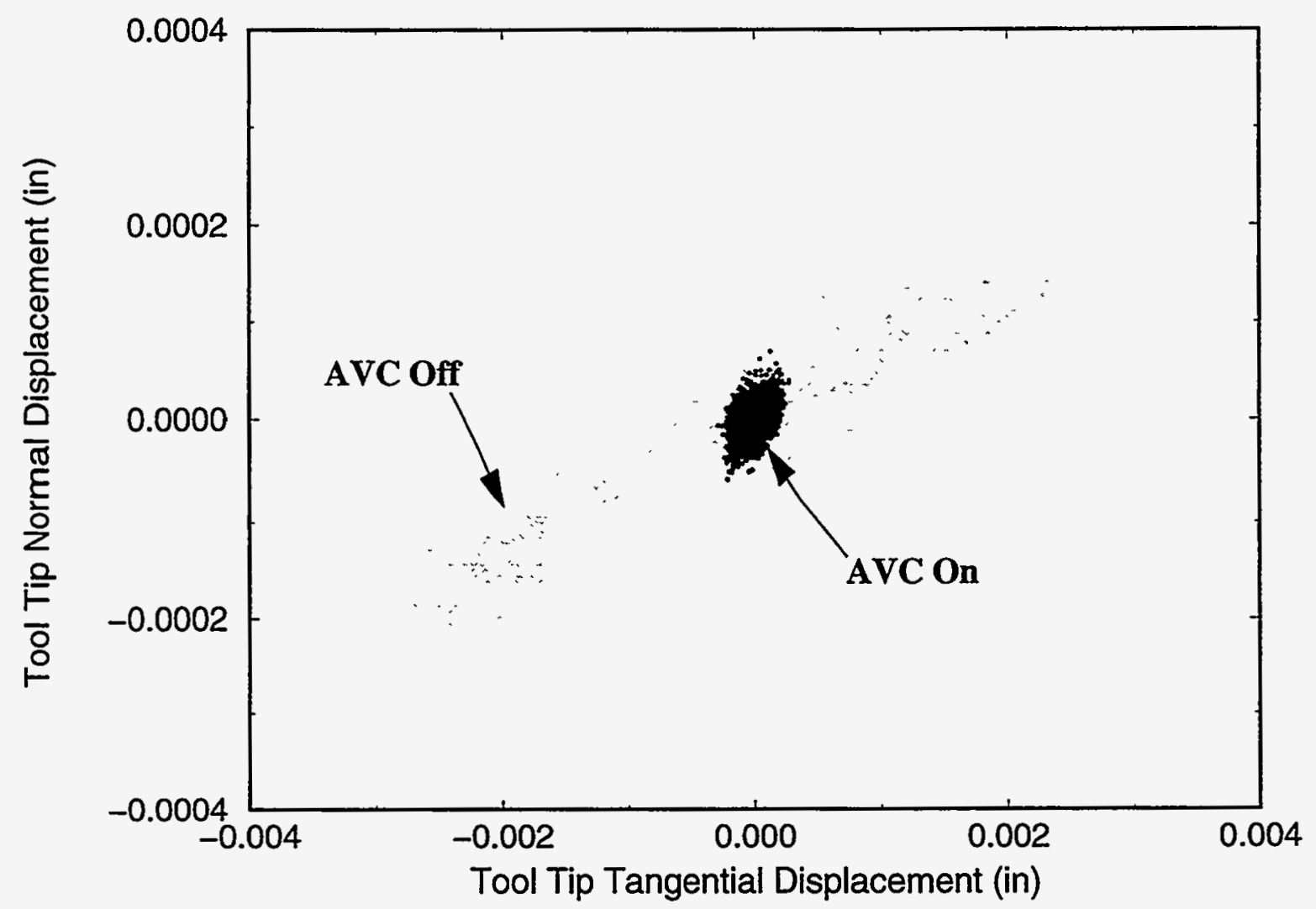

Figure 5.8. - Estimated Tool Tip Displacement Portrait for 0.005 inch Depth of Cut, 0.006 inches/rev Feed Rate, and 112 rpm.

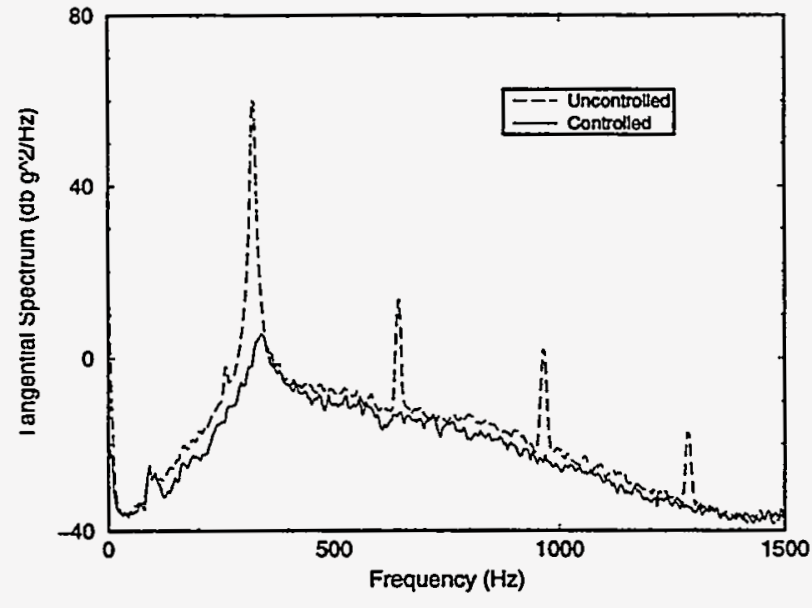

Figure 5.9. - Tangential Acceleration Spectrum for 10 mil Depth of Cut.

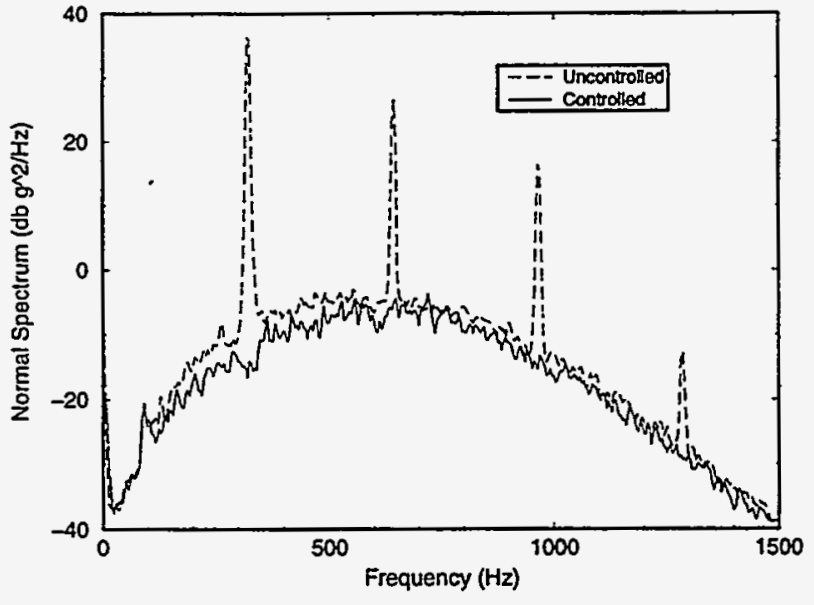

Figure 5.10. - Normal Acceleration Spectrum for 10 mil Depth of Cut.

transition period is evident during which the tool worked to overcome the forced vibration caused by the previously recorded chatter marks on the workpiece surface. By 2.5 seconds, the chatter was eliminated and stable machining occurred until the controller was shut down at 7 seconds. Again a transition period existed, during which the chatter slowly grew, covering more of the workpiece surface with each revolution. By nine seconds, the chatter covered the entire circumference of the workpiece, and was sustained at a high level until the cut was terminated. 


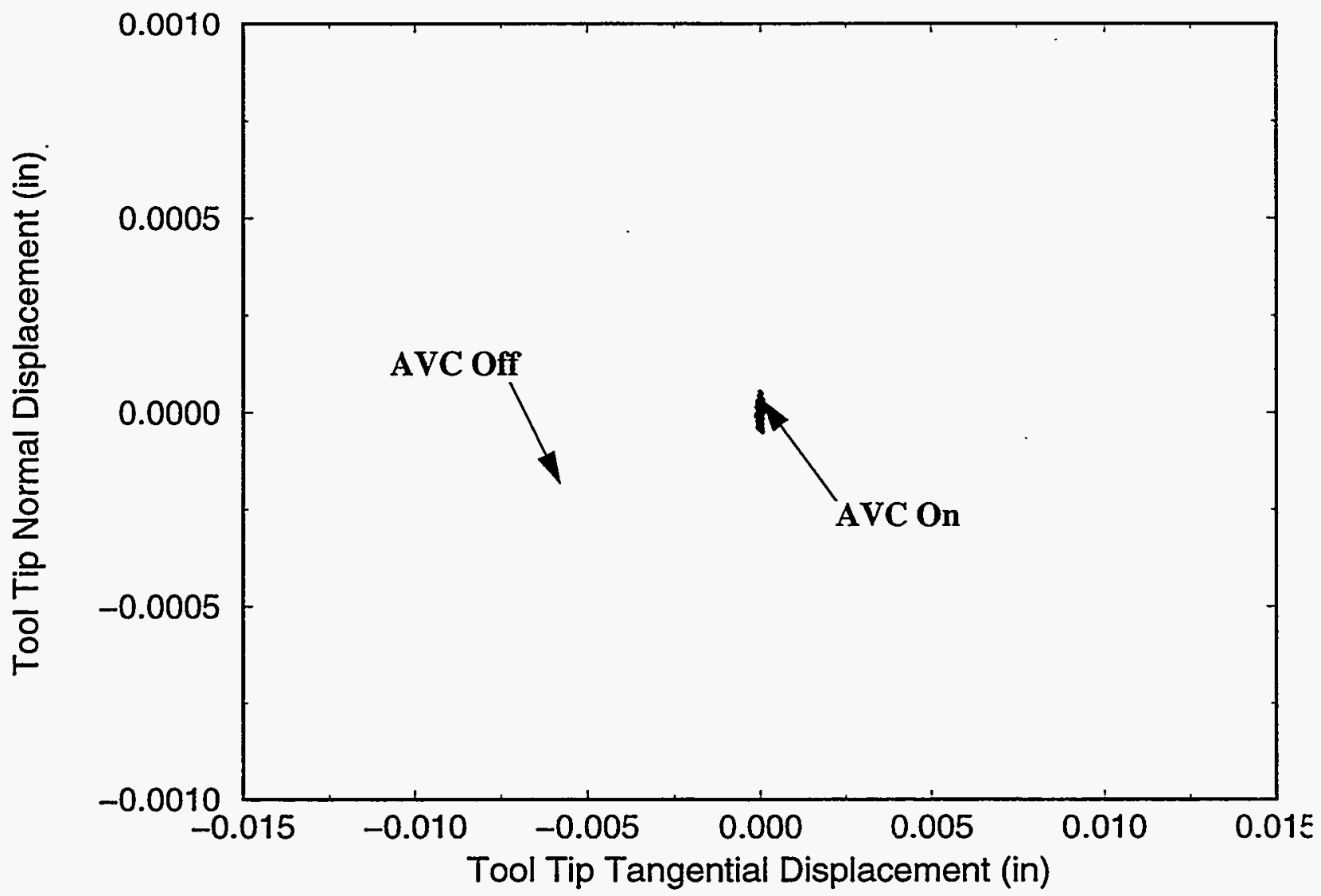

Figure 5.11. - Estimated Tool Tip Displacement Portrait for 0.010 inch Depth of Cut, 0.0045 inches/rev Feed Rate, and 127 rpm.

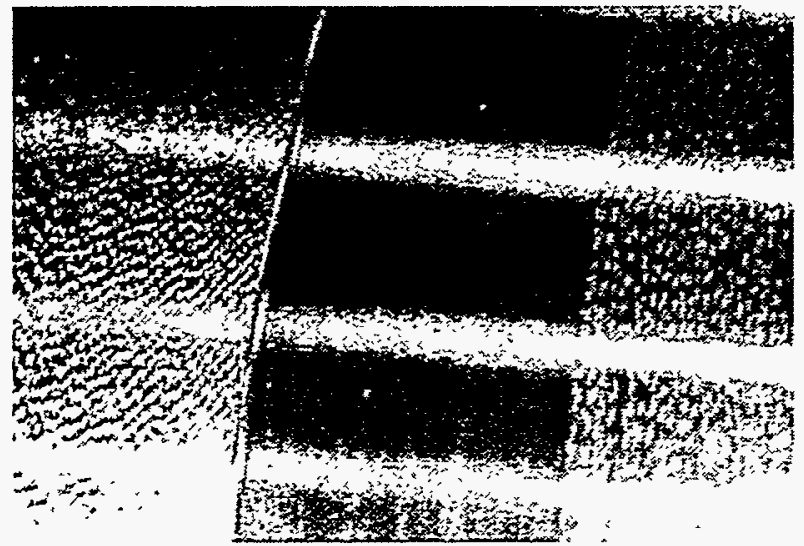

Figure 5.12. - Surface Finish Comparison for Sample 10 mil Depth of Cut.

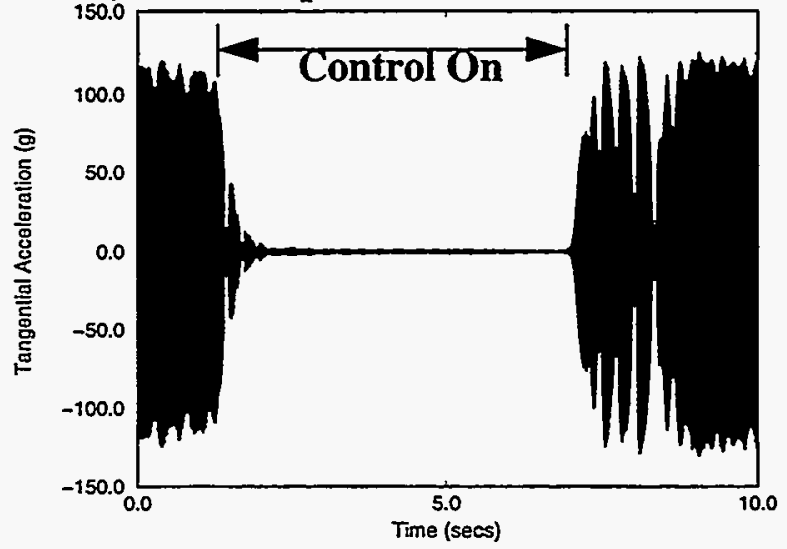

Figure 5.13. - Example of Off-On Control Transition for 10 mil Depth of Cut. 


\subsubsection{Depth of Cut 0.015 inches}

Power spectra for the case of 0.015 inch depth of cut at $160 \mathrm{rpm}$ with feed rate of 0.006 inches/ rev are shown in Figures 5.14 and 5.15. Again the uncontrolled case shows strong evidence for the presence of chatter. Although a small resonant peak remained after the application of AVC, the lack of harmonic peaks was indicative of a steady vibration not building to chatter. This is verified by the tool tip displacement portrait given in Figure 5.16. For this example surface roughness was reduced from 120 to $60 \mu$ inches.

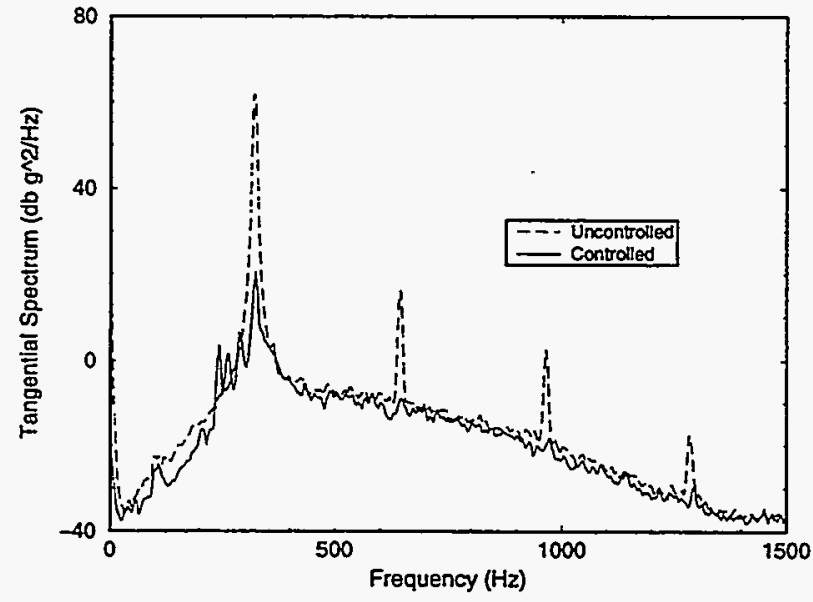

Figure 5.14. - Tangential Acceleration Spectrum for 15 mil Depth of Cut.

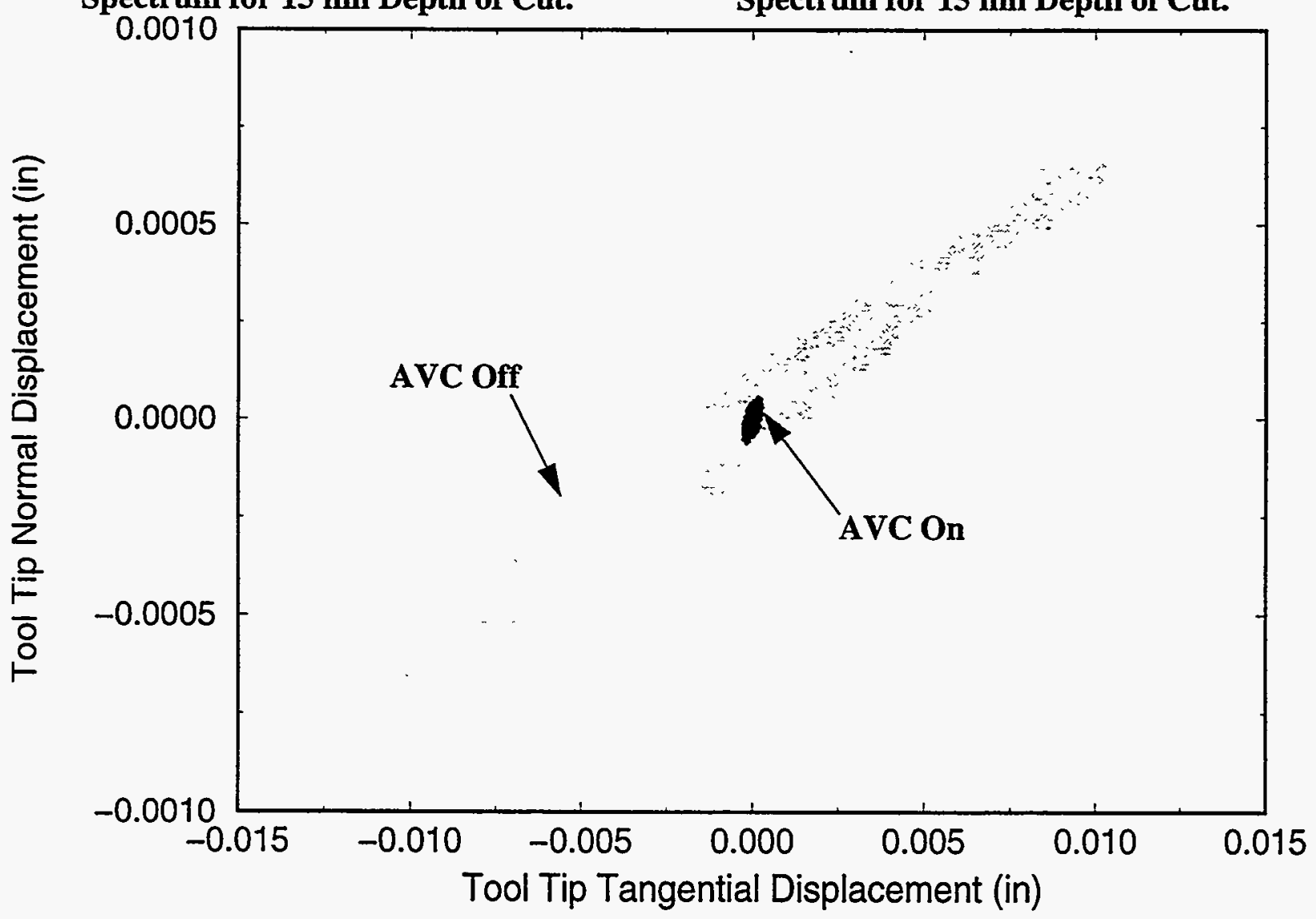

Figure 5.16. - Estimated Tool Tip Displacement Portrait for 0.015 inch Depth of Cut, 0.006 inches/rev Feed Rate, and 160 rpm. 


\subsubsection{Depth of Cut 0.020 inches}

As shown in Figures 5.17 and 5.18, sustainable chatter for a 0.02 inch cut depth was established with a workpiece speed of $79 \mathrm{rpm}$ and feed rate of 0.003 inches/rev. The chatter was again eliminated upon activation of the AVC, although the resonant peak dominates the residual vibration. The surface roughness for this example was reduced from $167 \mu$ inches to $85 \mu$ inches, owing to the reduced tool tip vibration amplitude shown in Figure 5.19.

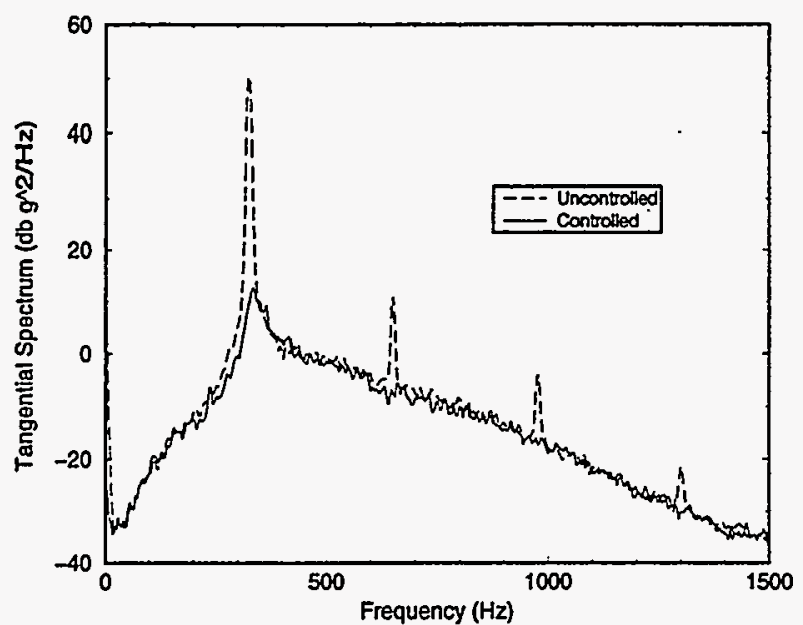

Figure 5.17. - Tangential Acceleration Spectrum for 20 mil Depth of Cut.

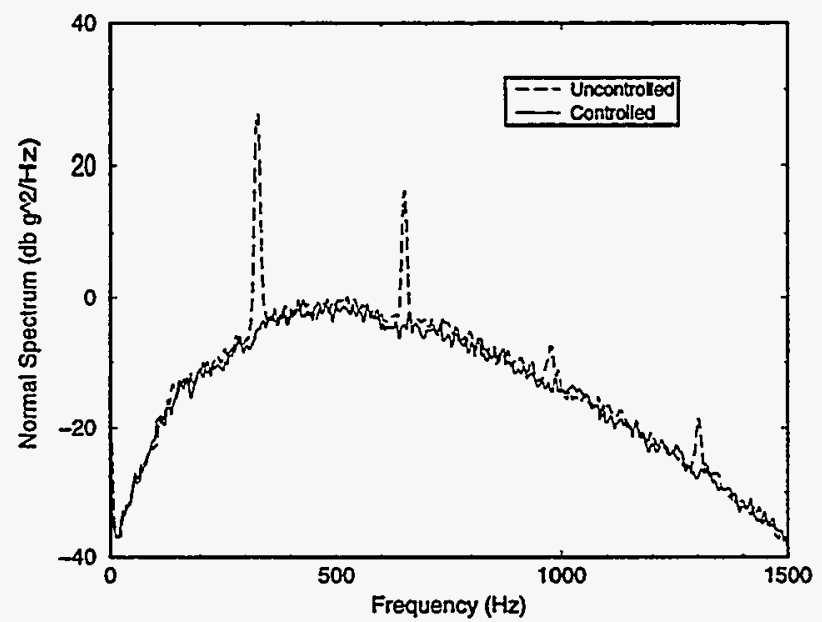

Figure 5.18. - Normal Acceleration Spectrum for 20 mil Depth of Cut.

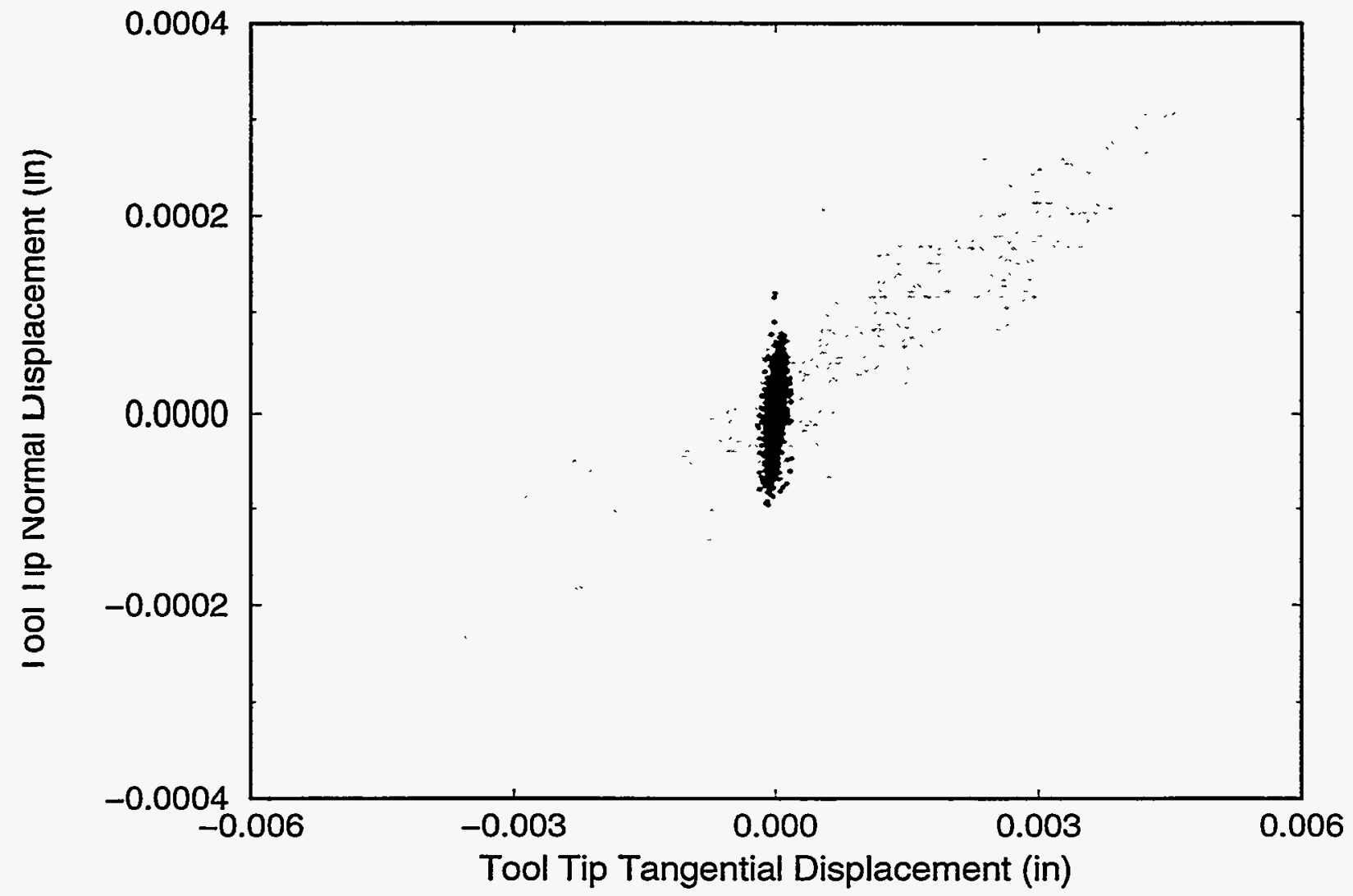

Figure 5.19. - Estimated Tool Tip Displacement Portrait for 0.020 inch Depth of Cut, 0.006 inches/rev Feed Rate, and 160 rpm. 


\subsection{Performance Assessment}

In the previous section, a number of sample cuts were examined to demonstrate the effectiveness of the actively damped boring bar in eliminating chatter. However, these examples provided little insight into the overall performance enhancement resulting from AVC. As previously mentioned, in many cases AVC can prevent chatter from occurring even when it is ineffective at removing chatter once it is sustained due to the stroke limitations of the actuators. Thus, it is necessary to scope out the chatter prevention capabilities of the actively controlled bar and compare them to a standard bar with no modifications.

Toward that end, a test matrix was contrived to explore vibration amplitude as a function of speed and feed for both the actively controlled bar and a pristine bar of identical make and model. With the depth of cut fixed at 0.015 inches, the workpiece speed was varied from 75 to $180 \mathrm{rpm}$ in 10 increments and the feed rate was swept from 0.0015 inches to 0.0195 inches in 13 increments. At each point on the test matrix, ten seconds of normal and tangential tool tip accelerations were recorded after a steady state vibration level was achieved.

Figures 5.20-23 contain approximate rms tool-tip displacements for the test matrix. Displacements were obtained from a narrow band integration of the raw acceleration data taken at the fundamental frequency. While this approach produces reasonably accurate results in the presence of chatter vibrations, significant magnitude errors can occur for low level vibrations that are not dominated by a signal frequency. This discrepancy is verified by comparing results of the test matrix to the broad band integration used on the individual cutting examples of the previous section. For this series of tests, however, narrowband integration was favored because it enabled rapid progression through the test matrix without overly burdening the storage capacity of the data aquisition system. Consequently, while results may not accurately reflect the magnitude of the lower level vibrations, the plots give a clear indication of the border between the stanble and unstable regions.

For the unmodified bar shown in Figures 5.20 and 5.22, chatter vibrations dominated the test matrix with the tangential and normal vibrations amplitudes on the order of 0.010 and 0.001 inches, respectively. Although a small region of chatter free machining was encountered at the highest speeds and feed rates, these conditions are characterized by poor surface finishes. In contrast to the unmodified bar, the bar with AVC yielded greatly reduced vibration levels over most of the parameter space considered as shown in Figures 5.21 and 5.23. Only the lowest speeds with the highest feed rates produced appreciable vibration levels. Thus, for the conditions considered, the modified bar drastically outperformed the unmodified bar and enabled the production of workpieces with acceptable surface finishes. 

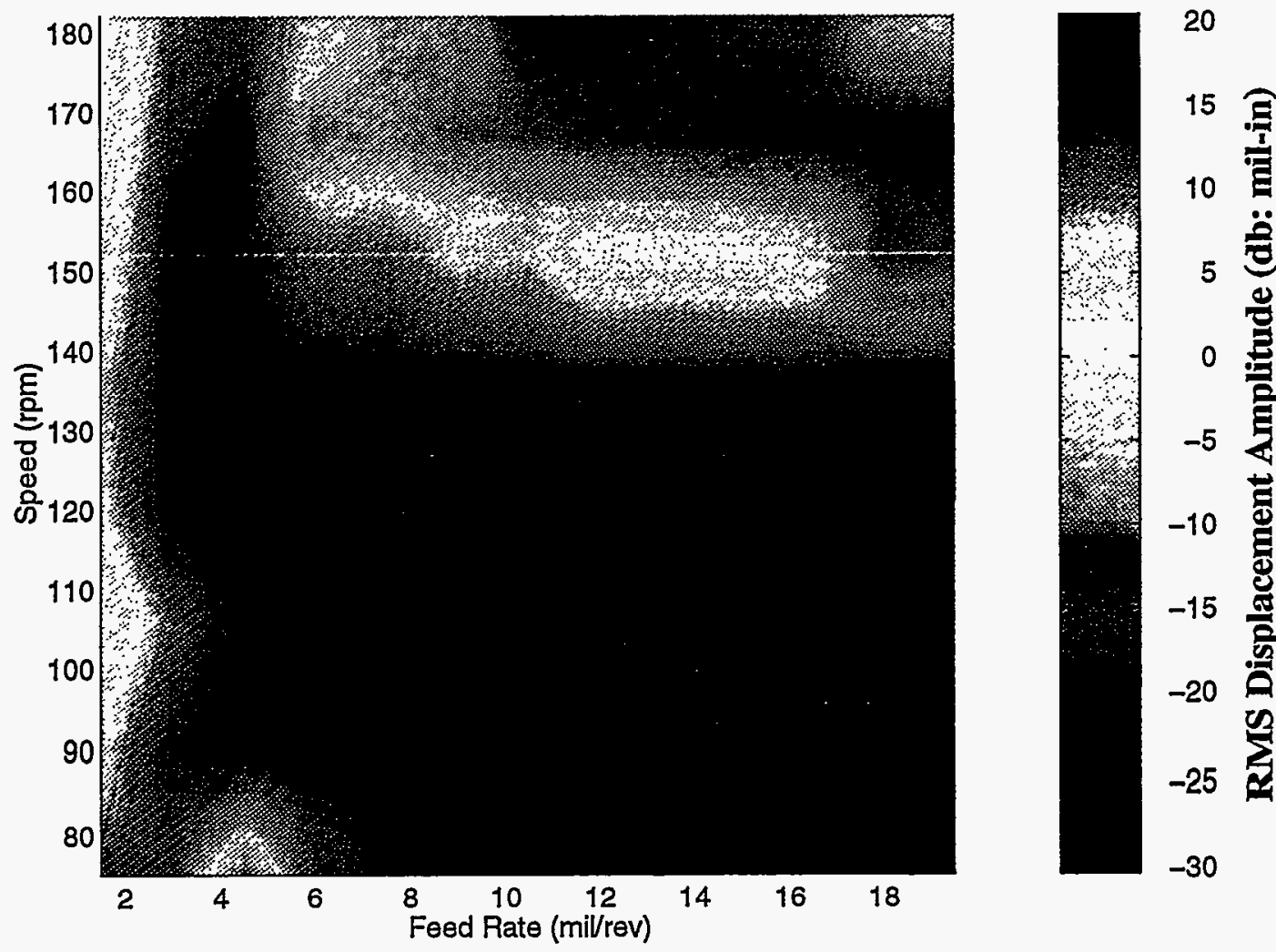

Figure 5.20. - Estimated Tangential Vibration Amplitude Contours for Unmodified Bar.
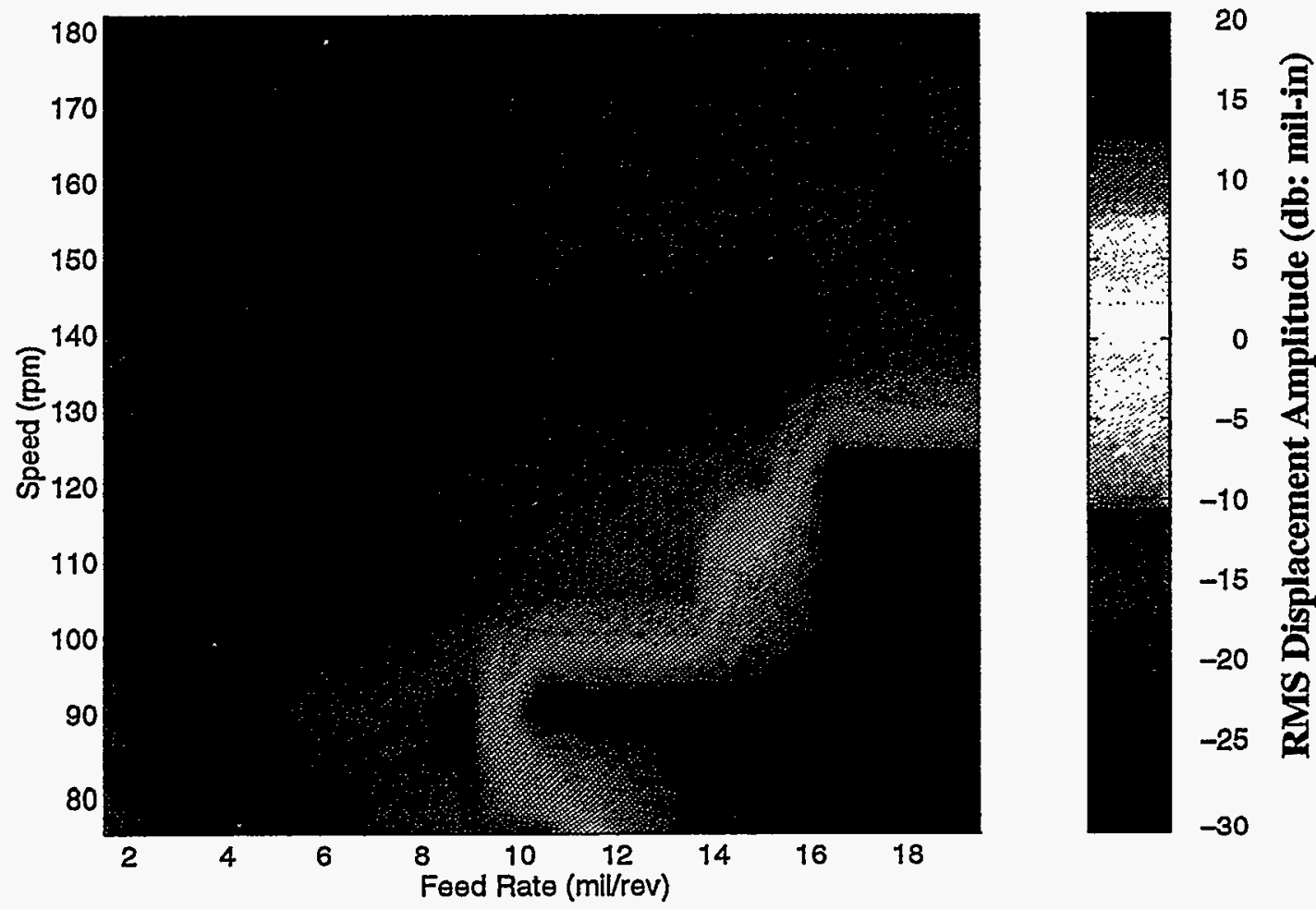

Figure 5.21. - Estimated Tangential Vibration Amplitude Contours for Controlled Bar. 

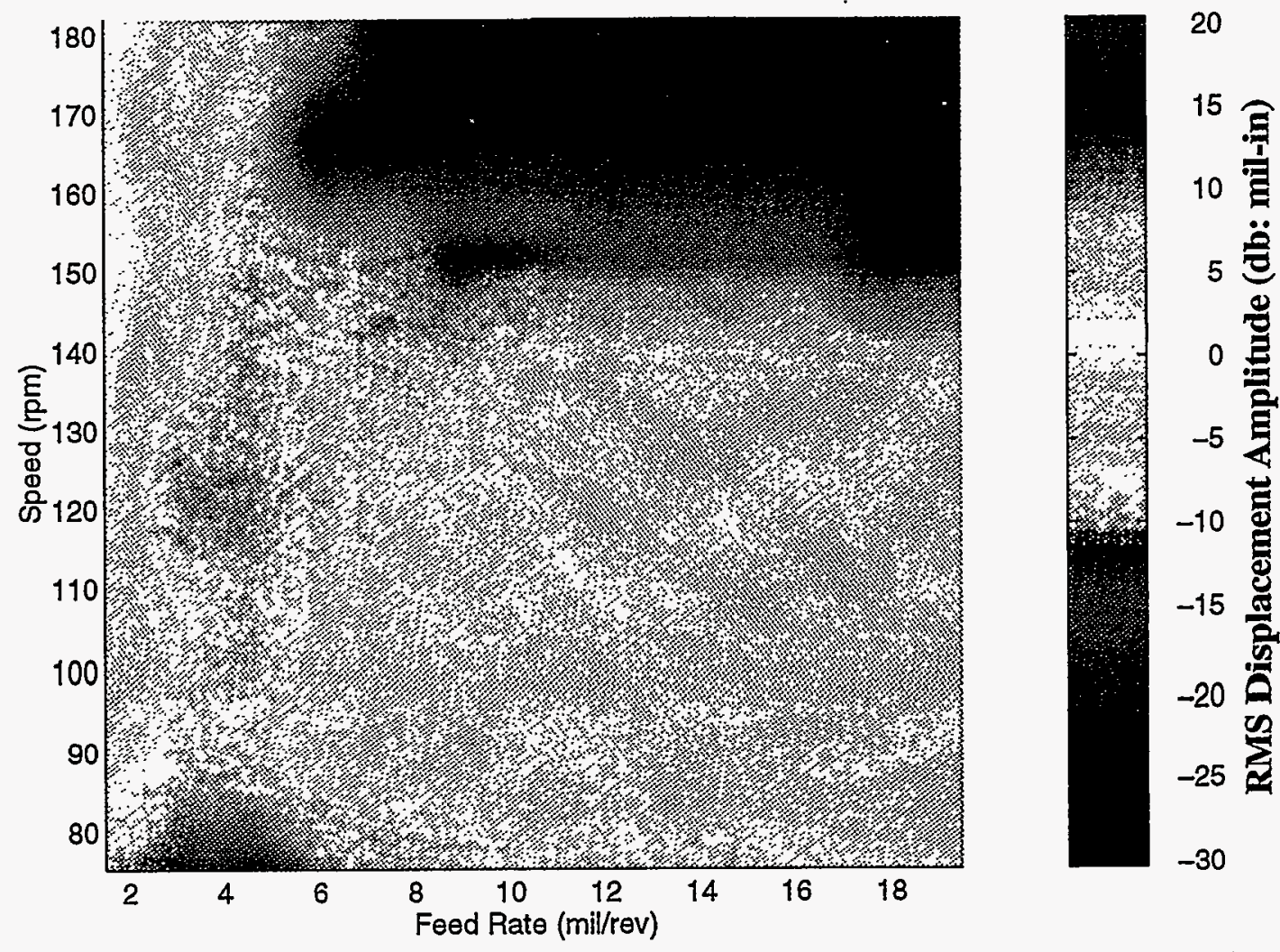

Figure 5.22. - Estimated Normal Vibration Amplitude Contours for Unmodified Bar.
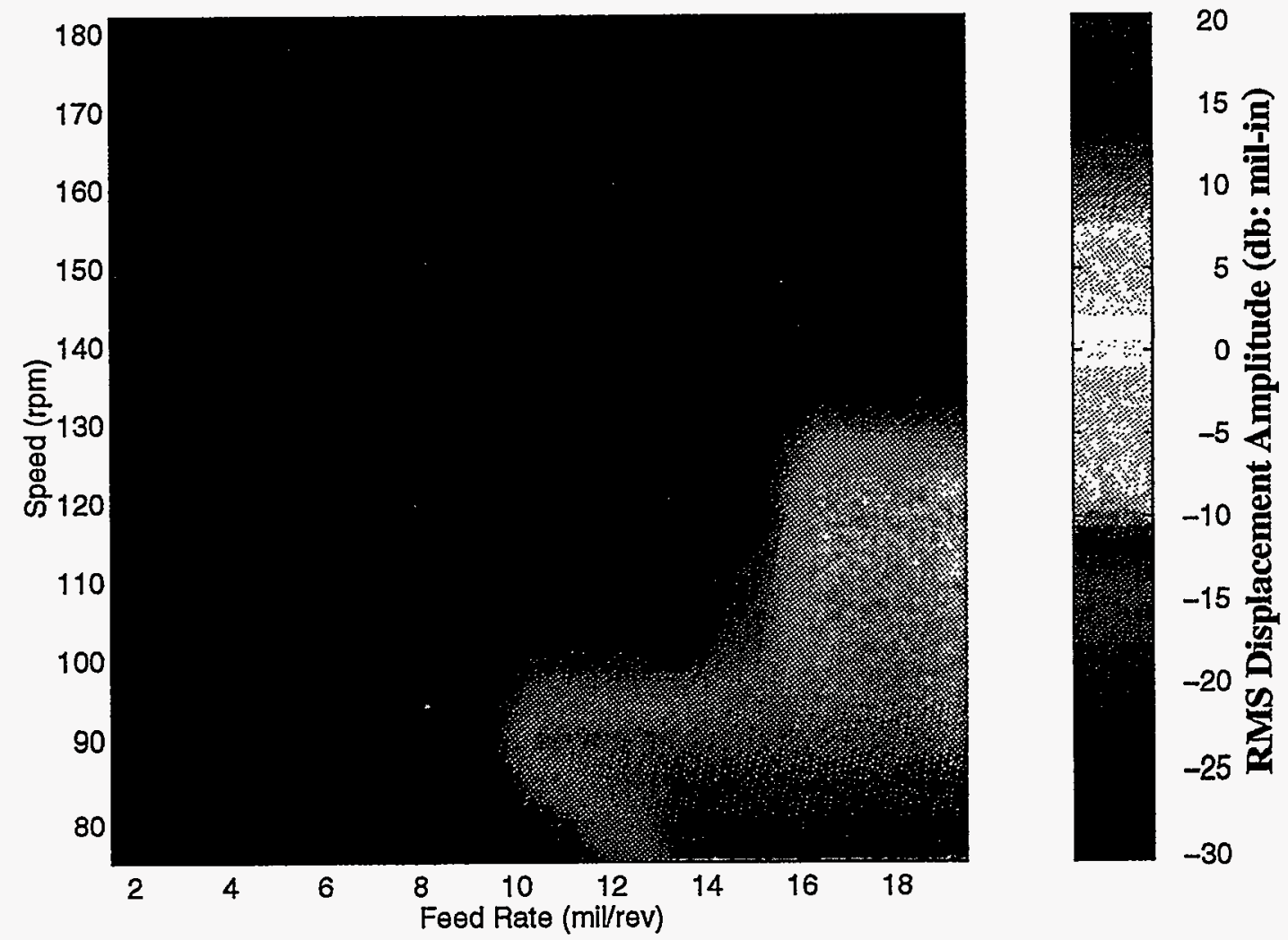

Figure 5.23. - Estimated Normal Vibration Amplitude Contours for Controlled Bar. 


\subsection{Concluding Remarks}

This project validated the concept of improving machining capabilities through the development of smart cutting tools. Two primary accomplishments include the development of a computer simulation to assess the performance of a cutting tool, and experimental validation of a prototype smart cutting tool.

In the first year of this two year project, a computer simulation of the boring process was developed to analytically evaluate the performance of a boring bar without conducting extensive cutting tests. Simple measurements of the boring bar's vibrational characteristics are used to form a dynamic model of the cutting tool. Cutting forces that drive the tool vibrations are determined by the instantaneous chip area, which depends on user selected machining parameters such as workpiece speed, tool feed rate, and depth of cut. Past tool vibration history leaves a waviness on the workpiece surface which also influences chip area, potentially leading to unstable regenerative chatter vibration. For simplicity, the chatter amplitude saturates when the tool vibration normal to the workpiece surface exceeds the cut depth. However, the significance of the tangential vibration in determining the saturation amplitude in practice was noted, and may be incorporated into future versions. Simulation output includes tool vibration histories normal and tangential to the workpiece surface, along with predicted cutting forces and an estimate of workpiece surface finish. A number of potential tool designs were evaluated using this simulation, leading to the development and testing of a prototype cutting tool.

Second year efforts focused on developing and testing prototype cutting tools with length to diameter ratios of 6,8 , and 10. Piezoelectric stack actuators were mounted in cutouts in the bar root to control bending vibrations induced by dynamic cutting forces. Closed-loop benchtop tests showed dramatic improvement in the dynamic characteristics of each bar, indicating the potential for significant enhancements to cutting performance. A series of cutting tests conducted at SNLALB building 840 on a Binns and Barry horizontal lathe validated the project hypothesis. Active vibration control (AVC) yielded a dramatic enlargement of the stable machining envelope for a bar with overhang of 6. For stable cuts, AVC produced a 2-3 time reduction in workpiece surface roughness as compared to an unmodified bar. Unfortunately, unanticipated project delays limited the time available for evaluation of the bars with overhangs of 8 and 10 , and only minimal data is available. However, the results obtained in bench top tests indicate that significant performance enhancements are available for these longer overhangs through the implementation of AVC. 


\subsection{References}

1. Merritt, H.E., "Theory of Self-Excited Machine Tool Chatter," Journal of Engineering for Industry, November, 1965, pp. 447-453.

2. Dohner, J.L.,Hinnerichs, T.D., Lauffer, J.P., Kwan, C.M., Regelbrugge, M.E., Shankar, N., "Active Chatter Control in a Milling Machine," SPIE-Vol. 3044, Proceedings of the Smart Structures and Materials 1997, San Diego, CA, March 4-6, 1997, PP. 281-294.

3. Tlusty, J., "Machine Dynamics", Handbook of High Speed Machining Technology, R.I. King ed., Chapman and Hall, New York, 1985.

4. Tewani, S.G., Rouch, K.E., and Walcott, B.L., "A Study of Cutting Process Stability of a Boring Bar With Active Dynamic Absorber," International Journal of Machine Tools, Vol. 35, No. 1, 1995, pp. 91-108.

5. Kim, K.J., and Ha, J.Y., "Suppression of Machine Tool Chatter Using a Viscoelastic Dynamic Damper," Journal of Engineering for Industry, Vol. 109, Feb. 1987, pp. 58-65.

6. Browning, D.R., Golioto, I., and Thompson, N.B., "Active Chatter Control System for Long Overhang Boring Bars," SPIE-Vol. 3044, Proceedings of the Smart Structures and Materials 1997, San Diego, CA, March 4-6, 1997, PP. 270-280.

7. Redmond, J.M., Parker, G.G., Barney, P.S., and Rodeman, R., "Active control of Bending Vibrations in Thick Bars Using PZT Stack Actuators," DSC-Vol. 57-2, Proceedings of the 1995 ASME International Mechanical Engineering Conference and Exposition, San Francisco, Ca, November 1995, pp. 831-835.

8. Matlab Reference Guide, The Matworks Inc., 1995.

9. Kapoor, S.G. DeVor, R.E., and Skarvan, R.D., An Enhanced Computer Based Process Simulation Model for the Cylinder Boring Process," Proceedings of the 42nd SAE Earthmoving Industry Conference, Peoria, II, April 9-10, 1991.

10. Dohner, J.L., "System Identification for Robust Control Design," Sandia Report, SAND950843, April 1975.

11. Merchant, M.E., "Basic Mechanics of the Metal-cutting Process," Journal of applied Mechanics, September 1944, pp. 168-175.

12. Smith, S., and Tlusty, J., "An Overview of Modeling and Simulation of the Milling Process," Journal of Engineering for Industry, Vol. 113, May 1991, pp. 169-175.

13. Drozda, T.J., and Wick, C., Tool and Manufacturing Engineers Handbook, Society of Manufacturing Engineering, 1983, pp. 14-15.

14. Redmond, J.M. and Barney, P.S., "Active Vibration Control of Stiff Beams and Plates Using Structurally Integrated PZT Stack Actuators," Journal of Intelligent Material Systems and Structures, Vol. 8, May 1998.

15. Chaudry, Z. and Rogers, C.A., "Enhanced Induced Strain Actuator Authority Through Discrete Attachment to Structural Elements," AIAA Journal, Vol. 31, No. 7, 1991, pp. 1287-1292.

16. Choe, K. and Baruh, H., "Actuator Placement in Structural Control," Journal of Guidance, Control, and Dynamics, Vol. 15, No. 1, 1992, pp. 40-48.

17. Baruh, H. and Tadikonda, S.S.K., "Gibbs Phenomenon in Structural Control," Journal of Guidance Control and Dynamics, Vol. 14, No. 1, 1991, pp. 51-58. 
18. Abaqus Reference Manual, Ver. 5.5, Hibbitt, Karlsson \& Sorensen, Inc., 1995.

19. Tlusty, J., "Machine Dynamics," Handbook of High Speed Machining Technology, R.I. King ed., Chapman and Hall, New York, 1985, pp. 49-153.

20. P. Barney, J. Redmond, and D. Smith, "Characteristics of Self-Sensing Actuation for Active Control," Proceedings of the International Modal Analysis Conference-XV, Orlando, FL, February 3-6, 1997. 


\section{Appendix: Publications Associated with The Smart Tool LDRD}

1. J. Redmond and P. Barney "Vibration Control of Stiff Beams and Plates Using Structurally Integrated PZT Stack Actuators," Journal of Intelligent Material Systems and Structures, Vol. 8 May 1998.

2. J. Redmond and P. Barney, "Development and Testing of an Actively Damped Boring Bar Featuring Structurally Integrated PZT Stack Actuators," submitted to the 9th CIMTEC, Florence, Italy, June, 1998.

3. J. Redmond, P.Barney, and D. Smith, "Development of an Active Boring Bar for Increased Chatter Immunity," SPIE's 4th Annual Symposium on Smart Structures and Materials, San Diego, CA, March 3-6, 1997.

4. P. Barney, J. Redmond, and D. Smith, "Characteristics of Self-Sensing Actuation for Active Control," International Modal Analysis Conference-XV, Orlando, FL, February 3-6, 1997. 
Distribution:

$\begin{array}{lll}\text { MS0318 } & 9215 & \text { G.S. Davidson } \\ \text { MS0321 } & 9200 & \text { W.J. Camp } \\ \text { MS0437 } & 9118 & \text { R.K. Thomas } \\ \text { MS0439 } & 9234 & \text { J.L. Dohner } \\ \text { MS0439 } & 9234 & \text { C.R. Dohrmann } \\ \text { MS0439 } & 9234 & \text { T.D. Hinnerichs } \\ \text { MS0439 } & 9234 & \text { D.R. Martinez } \\ \text { MS0439 } & 9234 & \text { J.M Redmond (15) } \\ \text { MS0439 } & 9234 & \text { D.J. Segalman } \\ \text { MS0441 } & 9226 & \text { R.W. Leland } \\ \text { MS0443 } & 9117 & \text { M.W. Heinstein } \\ \text { MS0443 } & 9117 & \text { H.S. Morgan } \\ \text { MS0507 } & 9700 & \text { K.G. McCaughey } \\ \text { MS0557 } & 9741 & \text { T.J. Baca } \\ \text { MS0557 } & 9741 & \text { P.S. Barney (15) } \\ \text { MS0557 } & 9741 & \text { J.P.Lauffer } \\ \text { MS0557 } & 9741 & \text { R. Rodeman } \\ \text { MS0561 } & 1481 & \text { E.D. Apodaca } \\ \text { MS0561 } & 1481 & \text { T.K. Litts } \\ \text { MS0561 } & 1481 & \text { E.K. Mote } \\ \text { MS0561 } & 1481 & \text { T.M. Simpson } \\ \text { MS0561 } & 1481 & \text { C.D. Salazar } \\ \text { MS0819 } & 9231 & \text { J.S. Peery } \\ \text { MS0820 } & 9232 & \text { P. Yarrington } \\ \text { MS0841 } & 9100 & \text { P.J. Hommert } \\ \text { MS0958 } & 1484 & \text { L.F. Bieg } \\ \text { MS0959 } & 1401 & \text { R.C. Reuter } \\ \text { MS0960 } & 1400 & \text { J.Q. Searcy } \\ \text { MS0961 } & 1403 & \text { D.L. Plymale } \\ \text { MS1033 } & 1401 & \text { W.N. Sullivan } \\ \text { MS1109 } & 9224 & \text { A.L. Hale } \\ \text { MS1110 } & 9223 & \text { D.S. Greenberg } \\ \text { MS1110 } & 9222 & \text { D.E. Womble } \\ \text { MS1111 } & 9221 & \text { S.S. Dosanjh } \\ \text { MS1111 } & 9225 & \text { G.S. Heffelfinger }\end{array}$

\section{MS0188 4523 LDRD Office (5)}

MS9018 8940-2 Central Technical Files

MS0899 4916 Technical Library (5)

MS0619 12690 Review and Approval Desk,for DOE/OSTI (2)

MS0161 11500 Patent and Licensing Office 
Mr. David Smith

Machine Tool Research Center

Mechanical Engineering Department

University of Florida

Gainesville, Florida 32611

Dr. Jeff Abler

Precision Engineer

Precision Manufacturing/Manufacturing Technology

United Technologies Pratt\&Whitney

400 Main Street, MS 114-38

East Hartford, Connecticut 08108

Mr. Andy Hazelton

Nikon Research Corporation of America

1399 Shoreway Rd.

Belmont, CA 94002

Professor Gordon Parker

Michigan Technological University

815 R.L. Smith ME-EM Building

1400 Townsend Drive

Houghton, MI 49931

Dr. Erdal Unver

Lucent Technologies

1919 S. Eads Street Suite 300

Arlington, VA 22202

Mr. Doug Browning

Lucent Technologies

Bell Laboratories

Whippany, NJ 07981

Mr. Joe Kamman

Director, Corporate R\&D Tech Services

Department 97-B

4701 Marburg, Avenue

Cincinnati, OH 45209 